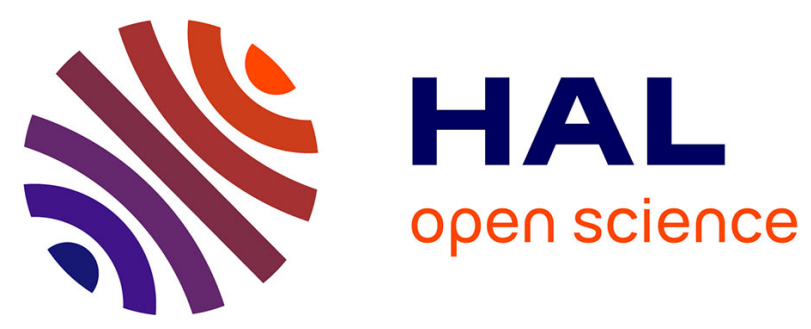

\title{
Drag and lift reduction of a 3D bluff-body using active vortex generators
}

\author{
Jean-Luc Aider, Jean-François Beaudoin, José Eduardo Wesfreid
}

\section{To cite this version:}

Jean-Luc Aider, Jean-François Beaudoin, José Eduardo Wesfreid. Drag and lift reduction of a 3D bluffbody using active vortex generators. Experiments in Fluids, 2010, 48 (5), pp.771-789. 10.1007/s00348009-0770-y · hal-01654042

\section{HAL Id: hal-01654042 \\ https://hal.science/hal-01654042}

Submitted on 2 Dec 2017

HAL is a multi-disciplinary open access archive for the deposit and dissemination of scientific research documents, whether they are published or not. The documents may come from teaching and research institutions in France or abroad, or from public or private research centers.
L'archive ouverte pluridisciplinaire HAL, est destinée au dépôt et à la diffusion de documents scientifiques de niveau recherche, publiés ou non, émanant des établissements d'enseignement et de recherche français ou étrangers, des laboratoires publics ou privés.

\section{(c)(1)}

Distributed under a Creative Commons Attribution| 4.0 International License 


\title{
Drag and lift reduction of a 3D bluff-body using active vortex generators
}

\author{
Jean-Luc Aider · Jean-François Beaudoin • \\ José Eduardo Wesfreid
}

\begin{abstract}
In this study, a passive flow control experiment on a 3D bluff-body using vortex generators (VGs) is presented. The bluff-body is a modified Ahmed body (Ahmed in J Fluids Eng 105:429-434 1983) with a curved rear part, instead of a slanted one, so that the location of the flow separation is no longer forced by the geometry. The influence of a line of non-conventional trapezoïdal VGs on the aerodynamic forces (drag and lift) induced on the bluffbody is investigated. The high sensitivity to many geometric (angle between the trapezoïdal element and the wall, spanwise spacing between the VGs, longitudinal location on the curved surface) and physical (freestream velocity) parameters is clearly demonstrated. The maximum drag reduction is $-12 \%$, while the maximum global lift reduction can reach more than $-60 \%$, with a strong dependency on the freestream velocity. For some configurations, the lift on the rear axle of the model can be inverted $(-104 \%)$. It is also shown that the VGs are still efficient even downstream of the natural separation line. Finally, a dynamic parameter is chosen and a new set-up with motorized vortex generators is proposed. Thanks to this active device. The optimal configurations depending on two parameters are found more easily, and a significant drag and lift reduction (up to $-14 \%$ drag reduction) can be reached for different freestream
\end{abstract}

J.-L. Aider · J.-F. Beaudoin

PSA Peugeot-Citroën, Research and Innovation Department, route de Gisy, 78943 Vélizy-Villacoublay, France

J.-L. Aider $(\bowtie) \cdot$ J.-F. Beaudoin · J. E. Wesfreid Laboratoire PMMH, UMR 7636, CNRS, ESPCI,

University Paris 6, University Paris 7-10, rue Vauquelin,

75231 Paris cedex 05, France

e-mail: aider@pmmh.espci.fr velocities. These results are then analyzed through wall pressure and velocity measurements in the near-wake of the bluff-body with and without control. It appears that the largest drag and lift reduction is clearly associated to a strong increase of the size of the recirculation bubble over the rear slant. Investigation of the velocity field in a crosssection downstream the model reveals that, in the same time, the intensity of the longitudinal trailing vortices is strongly reduced, suggesting that the drag reduction is due to the breakdown of the balance between the separation bubble and the longitudinal vortices. It demonstrates that for low aspect ratio 3D bluff-bodies, like road vehicles, the flow control strategy is much different from the one used on airfoils: an early separation of the boundary layer can lead to a significant drag reduction if the circulation of the trailing vortices is reduced.

\section{Introduction}

Flow control of separated and complex flows is a challenge in both academic and industrial research. From the industrial point of view, flow control is a way to increase the performance of a given vehicle (aeronautics, car manufacturer, naval industry) or of the production apparatus (chemical industry, energy production). From the academic point of view, it is an exciting theoretical and experimental problem implying a good knowledge of the target flow in order to choose the optimal perturbation to control the flow. The first step is then to define a control strategy to modify the flow in order to reach the chosen objective. If the objective is to reduce the drag or lift forces it is then important to identify the flow structures that contribute the most to the aerodynamics forces to be able to choose and to place properly the actuator. 
(a)

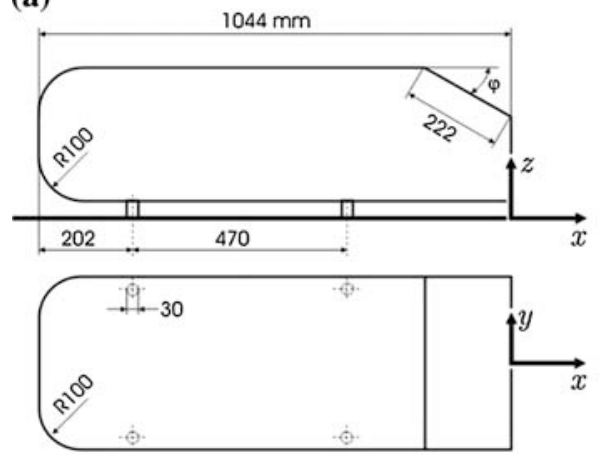

Fig. 1 a Side view and upper view of the original Ahmed body with a $30^{\circ}$ rear slant. b Schematic view of the rear of the model together with a sketch of the main flow structures expected over the rear of the Ahmed body with a rear slant angle $15^{\circ} \leq \theta \leq 30^{\circ}$ : recirculation

The strategy to control the flow over a road vehicle is very different from the one used to control the flow over an airfoil or the body of an airplane (Joslin 1998): the ground effect, the rotation of the wheels and the complex geometries lead to a fully unsteady and complex 3D flow (Hucho 1998). Moreover skin friction is negligible and the aerodynamic forces (especially drag and lift forces) are mainly governed by pressure losses. The consequence is that the control of wall turbulence (Bewley et al. 2001; Kim 2003) has rarely been tested in automotive aerodynamics compared to the control of separation and large coherent structures (Gad-El-Hak and Bushnell 1991; Greenblatt and Wygnanski 2000).

As it is difficult to deal with all the complexity of the flow over a real vehicle, it is important to define a simple 3D geometry to study the relation between the structures of the near-wake and the aerodynamic forces. The most famous bluff-body used in automotive aerodynamics is the socalled "Ahmed body" (Ahmed 1983), which has a blunt forepart and a rear part defined with different slant angles, flat panels and sharp edges (Fig. 1a). The forepart is designed to avoid separation so that the aerodynamic forces are mainly governed by the large vortical structures created on the rear part of the bluff-body (Fig. 1b): a closed or open separation bubble over the rear slant (for slant angle $15^{\circ} \leq \theta \leq 30^{\circ}$ ), a torus on the base of the rear part and two longitudinal vortices created on the side edges of the rear slant (the so-called "C-pillar vortex" in automotive aerodynamics). The example of the $30^{\circ}$ rear slant has been chosen to illustrate the discussion because, even if it is highly unstable, there is a competition between the main flow structures one can expect over the rear of a 3D bluffbody, unlike the $\theta<15^{\circ}$ or the $\theta>30^{\circ}$ where either the rear slant bubble or the longitudinal vortices are missing. Here, one should emphasize that our description is a

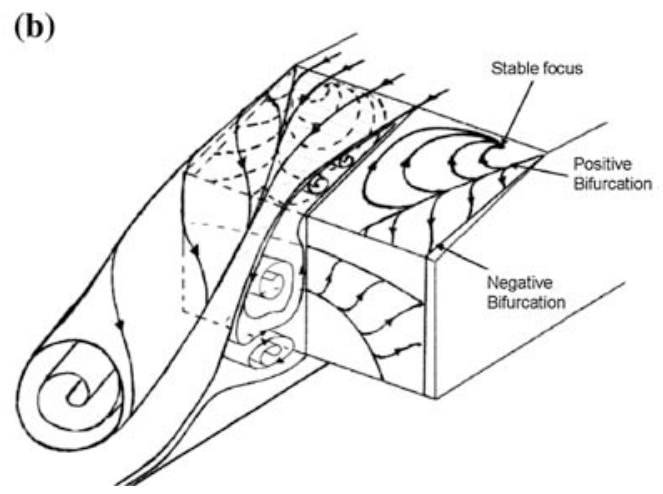

bubble, longitudinal vortices and recirculation torus. This sketch is taken from an experimental study of Vino et al. (2005) on an Ahmed body with a $30^{\circ}$ rear slant and an a Reynolds number $\operatorname{Re}_{L}=2.8 \times 10^{6}$

simplification of the real topology of the flow as demonstrated by Spohn and Gilliéron (2002) for the $\theta=25^{\circ}$ configuration and by Vino et al. (2005) for the $\theta=30^{\circ}$ configuration, but it is sufficient for the following discussions. In particular, the unsteady characteristics of the flow would not be considered in order to focus on the timeaveraged structure of the near-wake. One of the main differences in the description of the flow given by Vino et al. (2005), compared to the one of Ahmed et al. (1984) is that the separated flow does not reattach over the rear slant leading to strong interaction with the recirculation torus as shown also by Gilliéron and Chometon (1999). One can also notice that this sketch shows vortex shedding in the recirculation bubble, which can be witnessed only in instantaneous visualizations or measurements. They are cancelled out by time-averaging, which may be an explanation for the fact that these spanwise vortices were not observed in other studies. Whatever the exact topology of the flow, one of the main interest of this geometry is that it reproduces the main features of the near-wake of a hatchback vehicle. It was also especially useful to demonstrate the influence of the rear slant angle on the near-wake structure and on the drag force. In the perspective of flow control, and more precisely boundary layer manipulation, the rear part of the Ahmed body has been modified. The new geometry is detailed in Sect. 2.

From a general point of view, one can distinguish many different strategies to control a separated flow. Depending on the configurations and objectives, one can:

- control the shear layer at the separation (Chun et al. 1999; Aider and Beaudoin 2008; Verzicco et al. 2002). It is easier when the location of the separation is well defined like in the case of the backward-facing step or the Ahmed body (Leclerc et al. 2006). 
- control the boundary layer upstream of the separation (Song and Eaton 2002), which is a less common strategy in automotive aerodynamics. It is interesting when the location of the separation is not geometrically imposed like in the case of a smoothly contoured ramp (Duriez et al. 2006). The interest, and complexity, of such a strategy is that controlling the upstream boundary layer will modify both the location of the separation and the properties of the shear layer.

- control the flow using actuation along the wall downstream the separation, like blowing, suction or both blowing and suction (synthetic jets). It can be efficient to control the separation (Roumeas et al. 2009), but the energy balance may be less favorable than its upstream counter-part.

- control the flow using actuation in the volume downstream the separation. For instance, it has been demonstrated both experimentally (Strykowski and Sreenivasan 1990; Dalton et al. 2001) and theoretically (Giannetti and Luchini 2007) that it is possible to modify the structure of the near wake of cylinder of diameter $d$ using a smaller cylinder (typically $d / 10$ ).

The objective of the present study is to modify the boundary layer properties using vortex generators to control the separated flow over the rear part of a 3D bluffbody. There are many ways to produce longitudinal vortices leading to a large set of mechanical or fluidic vortex generators that could be appropriate (Betterton et al. 2000; Smith 1994). In this study, an original vortex generator geometry is proposed and will be discussed later in the paper.

The parameters defining the VGs have a strong influence on their efficiency. This is the reason why a parametric study has been carried out to show the sensitivity of the drag and lift to the different parameters. Another objective is also to find one geometric parameter that could be used as a dynamic parameter in a closed-loop experiment (Beaudoin et al. 2006).

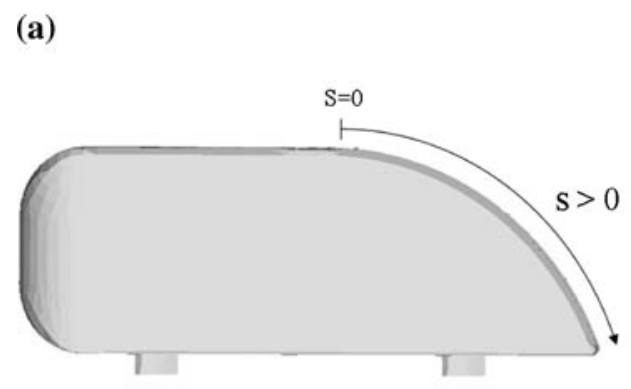

Fig. 2 On the left (a) side view of the bluff-body used in this study. The front part (on the left of the picture) is similar to the original Ahmed body while the rear part has a constant radius cross-section $(0.45 \mathrm{~m})$ in order to create an unsteady separation line. The origin of
The paper is organized as follows. In the first section, the experimental set-up and the 3D bluff-body are described. In the following sections, the vortex generators geometry and the corresponding parameters are presented before turning to the results of a detailed parametric study. The first results obtained with motorized VGs is also presented. It allows an easier two-parameters study leading to a global representation of the aerodynamic forces as a function of these parameters. An experimental investigation of the near-wake of controlled and uncontrolled bluffbody is then presented to try to understand the mechanisms associated to the drag and lift reduction. The last section is the conclusion.

\section{Experimental set-up and reference flow}

\subsection{Description of the bluff-body}

In order to deal with a 3D separated flow with a free separation line, the rear of the original Ahmed bluff-body (Ahmed et al. 1984), as shown on Fig. 1a, has been modified. The front part is unchanged (Fig. 2a), but the sharp edges and flat walls on the rear part are replaced by a rounded wall (Fig. 2b): the longitudinal cross-section of the rear part is now a constant radius circle arc. Thanks to this rounded slant, the separation line is no longer forced by the geometry. One can expect that the overall structure of the flow over the rear of the model should be a little different from the one of the Ahmed body shown on Fig. 1b: indeed, one can only expect a competition between the recirculation bubble induced by the separation over the rounded slant and the longitudinal vortices created along the side edges of the rear slant. At that point, it is convenient to introduce a curvilinear coordinate $s$ to define properly the location of the VGs over the rounded wall. Its origin $s=0$ is located at the beginning of the rounded wall and it is positive toward the rear of the model (Fig. 2a).

(b)

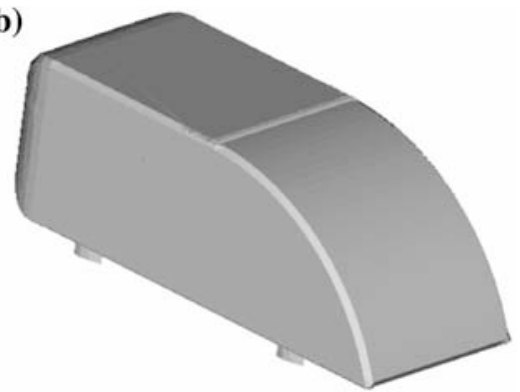

the curvilinear coordinate $s$ is at the beginning of the rounded wall and is positive toward the downstream direction. On the right (b) Upper view showing the $3 \mathrm{D}$ geometry 
Fig. 3 Description of the experimental facility. The crosssection of the wind tunnel is $5 \mathrm{~m}$ wide. The model is mounted over a raised floor so that the incoming turbulent boundary-layer is smaller than over the floor of the wind tunnel. The scales are not respected

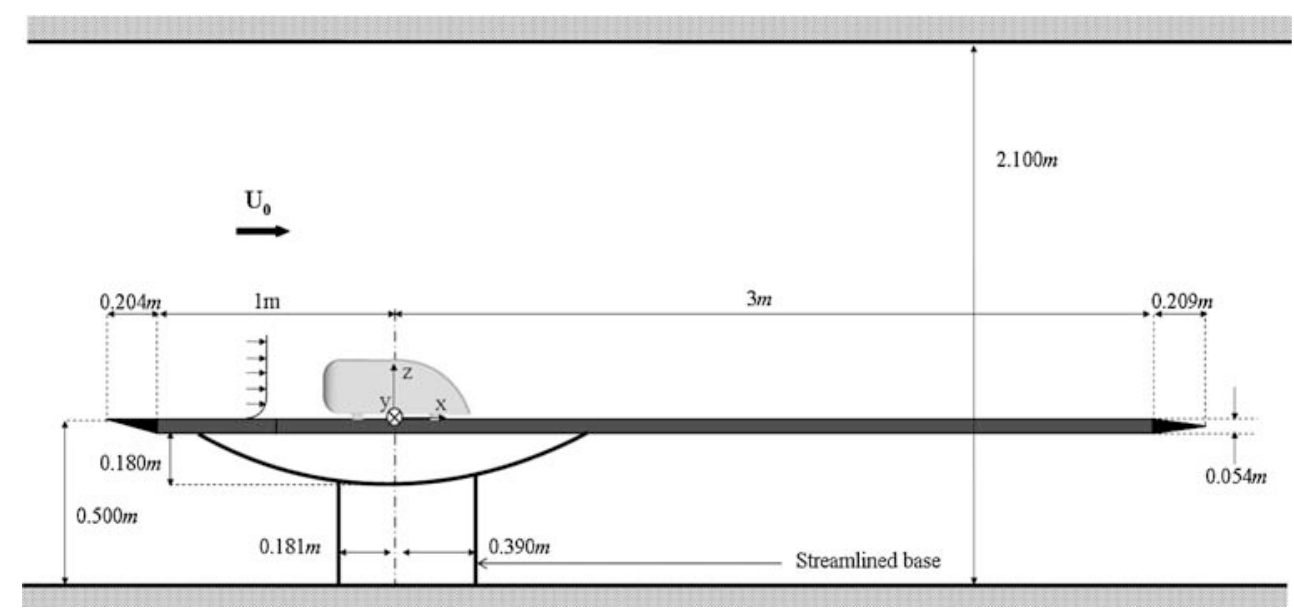

The model is $0.29 \mathrm{~m}$ high, $0.34 \mathrm{~m}$ wide and $0.90 \mathrm{~m}$ long. One should notice here that the dimensions are different from the original Ahmed body. The objective was to modify the original dimensions to be closer to the dimensions of a modern quarter-scale small vehicle (typically a 206 Peugeot at the time of the study). The curvature radius of its rear slant is $0.45 \mathrm{~m}$. There are two other differences with the classic Ahmed body: the height of the underbody is smaller than the one of the Ahmed body $(0.04 \mathrm{~m}$ instead of $0.05 \mathrm{~m})$ to be closer to realistic configurations, and the struts are profiled to minimize the perturbations induced by the the original rounded struts. One should mention that the same model has recently been used in the framework of a flow control experiments with flaps (Aider and Beaudoin 2008). The major difference is that the rear of the model was a flat slanted rear panel similar to the Ahmed body. The overall dimensions were the same as the ones of the model used in this study. Some information about the near-wake structure and aerodynamic coefficients of this more classic configuration can be found in this reference.

\subsection{Wind tunnel}

All the measurements are carried out in the PSA PeugeotCitroën in-house open wind tunnel (located in La Ferté Vidame, in France) which has a $6 \mathrm{~m}$ long closed test section, with a rectangular cross-section $2.1 \mathrm{~m}$ high and $5.2 \mathrm{~m}$ wide. The main characteristics of the flow in the wind tunnel are the following:

- free-stream velocity ranging from $U_{0}=20 \mathrm{~m} \mathrm{~s}^{-1}$ to $U_{0}=40 \mathrm{~m} \mathrm{~s}^{-1}$

- zero yaw angle

- Reynolds number $\operatorname{Re}=\frac{U_{0} L}{v}=1.2 \times 10^{6}$ to $2.4 \times 10^{6}$, $L$ being the length of the model

- turbulence intensity $=1.3 \%$
The coordinate axis are the following: $x$ is the streamwise direction and is positive downstream, $y$ is the spanwise direction and is positive left, while $z$ is the vertical direction and is positive upward, as it is the convention in automotive aerodynamics. The axis system origin is located on the ground at mid-wheelbase and mid-track. The velocity components $(u, v, w)$ are then defined respectively along the $(x, y, z)$ axis.

As can be seen in Fig. 3, a fixed raised floor was used for the measurements. The interest of such a configuration is to control the boundary layer thickness. It can be seen as an alternative to a boundary layer suction device. The raised floor is $3 \mathrm{~m}$ wide and $0.052 \mathrm{~m}$ thick. The overall blockage coefficient, including the bluff-body, the raised floor and its profiled struts, is about $5 \%$. The leading edge of the raised floor has been covered with sandpaper to avoid separation and generate a turbulent boundary layer over the raised floor. It has been checked through visualization and hot-wire velocimetry. The boundary layer thickness $\delta$ (reached when $u=0.99 \times U_{o}$ ) upstream of the model quickly decreases with tunnel speed: $\delta=6 \times$ $10^{-2} \mathrm{~m}$ for $U_{o}=20 \mathrm{~m} \mathrm{~s}^{-1}, \delta=3.5 \times 10^{-2} \mathrm{~m}$ for $U_{o}=30 \mathrm{~m} \mathrm{~s}^{-1}$ and $\delta=1.6 \times 10^{-2} \mathrm{~m}$ for $U_{o}=40 \mathrm{~m} \mathrm{~s}^{-1}$. A detailed analysis of the boundary layer over the raised floor can be found in Golhke et al. (2008) where it is clearly shown that the boundary layer is turbulent.

\subsection{Experimental measurements}

\subsubsection{Aerodynamic balance}

To evaluate the efficiency of the VGs on the aerodynamic forces, a six-components aerodynamic balance is used. Only the results on the drag and lift forces will be discussed. $C_{d}$ and $C_{l}$ are respectively the global drag and lift coefficients, while $C_{l \text { Rear }}$ is the lift coefficient applied on the rear-axle. They are defined as: 
Table 1 Reference values of the drag, global lift and rear-axle lift of the bluff-body for the three freestream velocities

\begin{tabular}{llll}
\hline$U_{o}\left(\mathrm{~m} \mathrm{~s}^{-1}\right)$ & $C_{d}$ & $C_{l}$ & $C_{\text {lRear }}$ \\
\hline 20 & 0.315 & 0.460 & 0.193 \\
30 & 0.291 & 0.396 & 0.147 \\
40 & 0.278 & 0.352 & 0.117 \\
\hline
\end{tabular}

$C_{d}=\frac{F_{x}}{\frac{1}{2} \rho S U_{0}^{2}}, C_{l}=\frac{F_{z}}{\frac{1}{2} \rho S U_{0}^{2}}$ and $C_{l \text { Rear }}=\frac{F_{z \text { Rear }}}{\frac{1}{2} \rho S U_{0}^{2}}$

where $F_{x / z}$ is the force in the $x$ or $z$ direction, and $S$ is the projected area of the model on the transverse plane. $F_{z \text { Rear }}$ is the lift force on the rear axle of the model. From the engineer point of view, $F_{z \text { Rear }}$ is more important than the global lift as it will be responsible for the loss of stability of vehicles in zero yaw and high speed situations. In the following, only the global lift coefficient will be discussed. Nevertheless, the rear axle lift will be mentioned in tables summarizing the aerodynamic coefficients. The typical error on the measurement of the aerodynamic forces is less than $1 \%$.

In Table, 1 the evolution of the drag and lift of the bluffbody for $U_{0}=20 \mathrm{~m} \mathrm{~s}^{-1}, U_{0}=30 \mathrm{~m} \mathrm{~s}^{-1}$ and $U_{0}=40 \mathrm{~m}$ $\mathrm{s}^{-1}$ are summarized. As expected, one can see a decrease of the drag and lift coefficients when the freestream velocity (Reynolds number) is increased. One can notice here that the drag coefficient of the bluff-body for $U_{0}=40 \mathrm{~m} \mathrm{~s}^{-1}$ is close to the one of the Ahmed body with a rear slant angle $\theta \simeq 25^{\circ}$ (Ahmed et al. 1984).

\subsubsection{Wall pressure measurements}

The static wall pressure coefficients $C_{p}$ are defined by Eq. 2:

$C_{p}=\frac{P-P_{0}}{\frac{1}{2} \rho U_{0}^{2}}$ where $U_{0}$ and $P_{0}$ are respectively the free-stream velocity and pressure measured upstream of the model, and $\rho$ is the density of the fluid. The pressure coefficients are measured on the vehicle body with $8 \times 10^{-4}$ m diameter pressure taps (Drück@). A total of 68 pressure taps are drilled through the vehicle wall. 62 sensors are located on the left half of the rear part of the model as shown in Fig. 4 (open circles over the curved wall), while the 6 remaining sensors are located on the other half in order to check the symmetry of the timeaveraged flow. Since the plane $y=0$ is actually a symmetry plane for the mean flow, the pressure coefficients distribution will be shown only on the left part. The data are recorded as long as necessary to reach a well converged mean value. The typical acquisition time for each probe is $10 \mathrm{~s}$.

\subsubsection{Particle image velocimetry set-up and base flow}

The Particle Image Velocimetry (PIV) set-up is used to measure two-components mean velocity field in different planes:

- $\quad(v, w)$ in the $x=$ const $=0.58 \mathrm{~m}$ plane,

- $(u, w) y=0$ plane,

- $(u, w) y=0.12 \mathrm{~m}$ plane,

- $(u, v) z=$ const $=0.15 \mathrm{~m}$ plane

Thanks to these different PIV measurements, one can identify the 3D structures of the flow. During the data acquisition, both laser source and camera are placed outside the test-section and the measurements are performed through transparent walls or sufficiently small holes to avoid perturbations (Fig. 5). The air-flow is seeded $12 \mathrm{~L}$ upstream from the model using a grid injection device, which is far enough not to perturb the incoming flow. In particular, the turbulent intensity is unchanged with or without the injection grid. Two Nd:Yag laser sources (120 mJ during a $10 \mathrm{~ns}$ pulse) provide double-pulsed light
Fig. 4 Distribution of the 62 pressure taps over the rear slant of the model together with the wall pressure field and the longitudinal velocity field obtained from PIV measurements in the vertical symmetry plane for the reference case with $U_{o}=20 \mathrm{~m} \mathrm{~s}^{-1}$
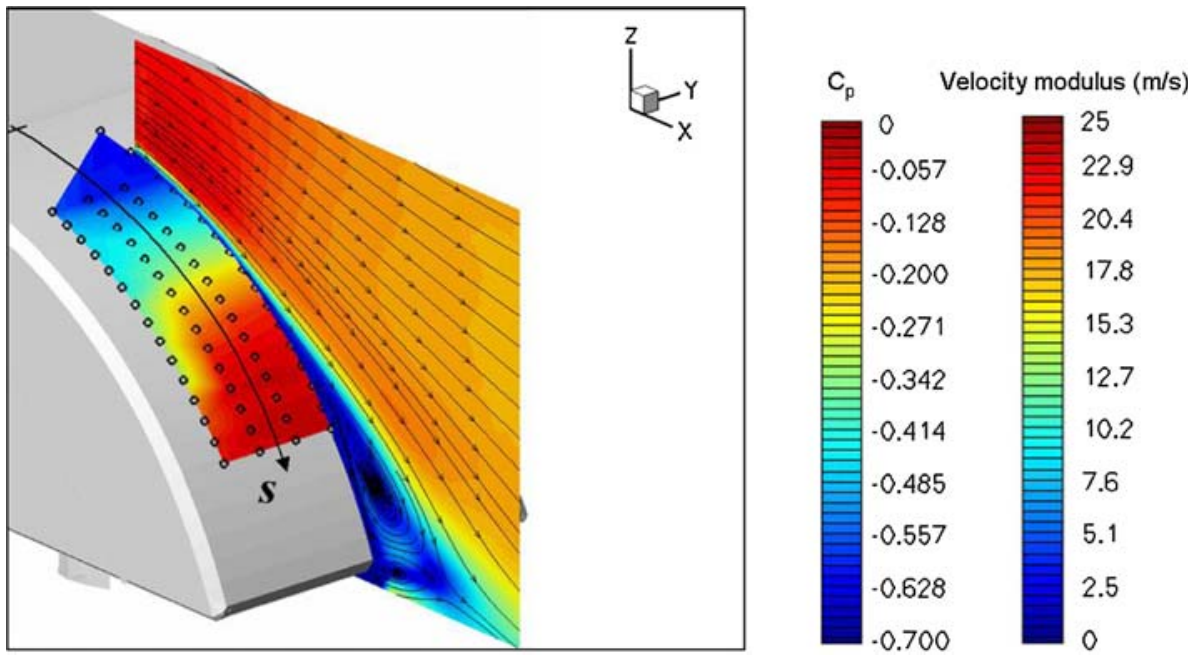


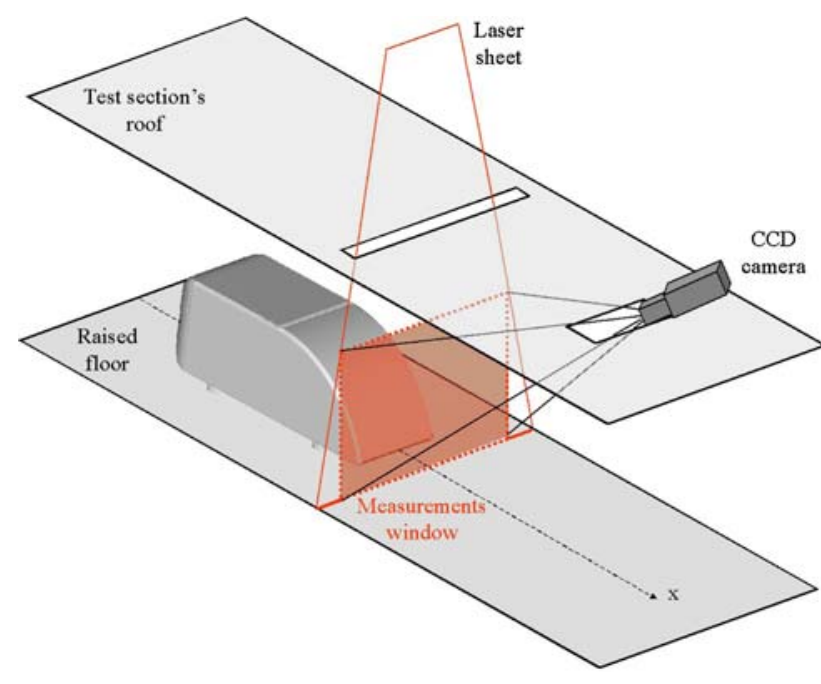

Fig. 5 PIV set-up used for measurements in transversal planes $(x=\mathrm{cst}=0.53 \mathrm{~m})$. The laser source and the CCD camera are placed above the test-section roof. The angle between the camera axis and the streamwise direction is relatively small $\left(30^{\circ}\right)$

sheets, which are approximately $3 \times 10^{-3} \mathrm{~m}$ thick. Images are recorded using a $1280 \times 1024$ pixels CCD camera with a $4 \mathrm{~Hz}$ acquisition rate. The typical physical dimensions of the PIV images are $0.4 \mathrm{~m} \times 0.5 \mathrm{~m}$. A $16 \times 16$ pixels interrogation window with a $25 \%$ overlap is used for the data-processing, leading to $4.8 \times 10^{-3} \mathrm{~m}$ spatial resolution. Two hundred and fifty instantaneous velocity fields are necessary to obtain the time-averaged wake of the bluff-body. The seeding particles are olive oil droplets in the range $1-10 \mu \mathrm{m}$. The size of the droplets is a good compromise to both follow the large-scale motions and be tracked by the PIV camera over a relatively large field. The droplets have also very satisfactory reflection properties.

To reduce the perturbations induced by the reflection of the laser beam on the wall of the model, a special fluorescent paint, FP R6G (Flow Visualization Component), is used. The paint absorb $99 \%$ of the laser light and reflects the remaining part at a $565 \mathrm{~nm}$ wavelength. An narrowband optical filter is then used on the video-camera filtering the wavelengths outside the range $532 \pm 5 \mathrm{~nm}$, and leading to a large reduction of the perturbations induced by the reflection of the laser light. Thanks to this set-up, one can see particles as close as $10^{-3} \mathrm{~m}$ from the wall.

A particular care is necessary for the PIV measurements in transversal planes $(x=$ const $)$ because particles may have a strong longitudinal velocity component. As a result, it is important to thicken the laser sheet (up to $5 \times 10^{-3} \mathrm{~m}$ ) and to shorten the time between two consecutive images (from $70 \mu \mathrm{s}$ in the case of $y=$ const or $z=$ const, to only $10 \mu \mathrm{s}$ in the case $x=$ const) in order to ensure that the particles remain in the laser sheet between two laser pulses. Moreover, a classic second order dewarping technique Raffel et al.
(2007) is applied in this particular case to take into account the $30^{\circ}$ angle between the camera axis and the streamwise direction (Fig. 5). No Scheimpflug optics was used so that there is no corrections for the out-of focus particles. Some errors can be expected due to the projection of the streamwise component on the local viewing angle, especially in the upper part of the picture where the streamwise velocity is larger. As the streamwise velocity is not constant over the cross-section, it is impossible to evaluate the error precisely. Moreover, in the following, one will only discuss the modification of the intensity of the trailing vortices which are low streamwise velocity regions so that one can consider that the expected error does not modify our conclusions about the modification of the structure of the near-wake.

As shown in Fig. 17a, b, the flow around the model exhibits the main features of automotive aerodynamics, since one can see both the recirculation torus on the base of the vehicle in the symmetry plane (Fig. 17a) and the longitudinal vortices arising on the sides of the rear part (Fig. 17b). These streamwise structures are low velocity regions (about $5 \mathrm{~m} / \mathrm{s}$ to be compared with $U_{0}=20 \mathrm{~m} / \mathrm{s}$ ). Their length is significantly larger than the size of the recirculation bubble. As shown by Beaudoin et al. (2004) through a cavitation experiment, the trailing vortices are also the lowest pressure regions in the near-wake, so that one can expect that they contribute significantly to the global drag of the bluff-body. One can notice that the overall structure of the near wake is different from the one shown on Fig. $1 \mathrm{~b}$ for a $30^{\circ}$ rear slant. In fact, the structure of the flow is closer to the $25^{\circ}$ rear slant configuration, i.e. a recirculation torus interacting with the trailing vortices.

Due to the three-dimensionality of the near-wake, the separation line is not parallel to the $y$ axis. From both PIV and pressure taps measurements, the position of the natural separation line could be estimated in terms of curvilinear coordinate (Fig. 2a): $s_{\text {nat }} \simeq 0.26 \mathrm{~m}$. Moreover, it is interesting to notice that the wall pressure is nearly uniform and reaches its highest value in the recirculation zone.

\subsubsection{Hot wire measurements}

A standard one component boundary-layer hot-wire probe (Dantec ${ }^{\odot}$ boundary layer probe $55 \mathrm{p} 15$ ) is used to investigate the boundary layer profile over the bluff-body. The single wire probe is moved over the bluff-body using a motorized device. Each velocity measurements is the average of $1.31 \times 10^{5}$ data with a $1 \mathrm{kHz}$ sampling frequency.

\section{Vortex generators for flow control}

The vortex generators used in this study are trapezoidal blades as shown in Fig. 6. The geometry is defined by four 


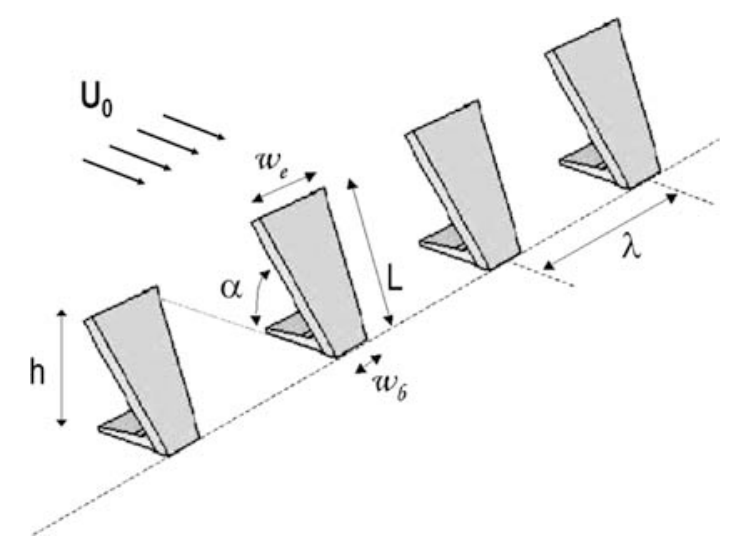

Fig. 6 Description of the VGs geometry with the typical dimensions characterizing the device used in these experiments. The angle between the blade and the wall, $\alpha$, is equal to $=60^{\circ}$ on this sketch but it is one of the parameters that will be varied in this study

parameters (Aider et al. 2003): the length $L$, base width $w_{b}$, and upper edge width $w_{e}$ of the trapezoidal blade and the angle $\alpha$ between the blade and the wall. The thickness of the blade is constant and equal to $10^{-3} \mathrm{~m}$. In most of the following study, the length $L$ of the VG will be $L=10^{-2}$ $\mathrm{m}$ and the angle $\alpha=60^{\circ}$ or $\alpha=120^{\circ}$, so that the apparent height of the VG is $h=L \sin (\alpha)=8.5 \times 10^{-3} \mathrm{~m}$.

The geometry of the VGs is rather unusual compared to classic vane type VGs used in aeronautics (Smith 1994; Betterton et al. 2000; Angele and Grewe 2002). The nature of the perturbations induced by the VG in a flat-plate boundary layer can not be easily guessed. As a matter of fact, it requires a detailed experimental study, which has been carried out in another experimental set-up. The results are detailed in a companion paper (Aider et al. 2009a), where it is shown that the vorticity is induced by the horseshoe vortex created around the base of the VG through a junction flow mechanism (Simpson 2001). The arms of horseshoe vortices interact with each other to finally create pairs of counter-rotating longitudinal vortices that will modify the properties of the boundary layer, depending on the spacing. A qualitative description is given in Fig. 7 where we show that a single trapezoidal blade creates one pair of counter-rotating streamwise vortices.

Another interest of this geometry is that the angle $\alpha$ between the blade and the wall on which the VG is mounted (Fig. 6) can be used as an active parameter, i.e. one can use $\alpha(I), I$ being an input in a closed-loop control. As will be shown in the following, one can design a motorized device where the input parameter $I$ could be the freestream velocity $U_{o}$ or the speed of the vehicle, in the case of an application on a real car (Aider et al. 2003). Such an application has been achieved on a Citroën concept-car called C-Airlounge (see Aider et al. 2009b and (a)
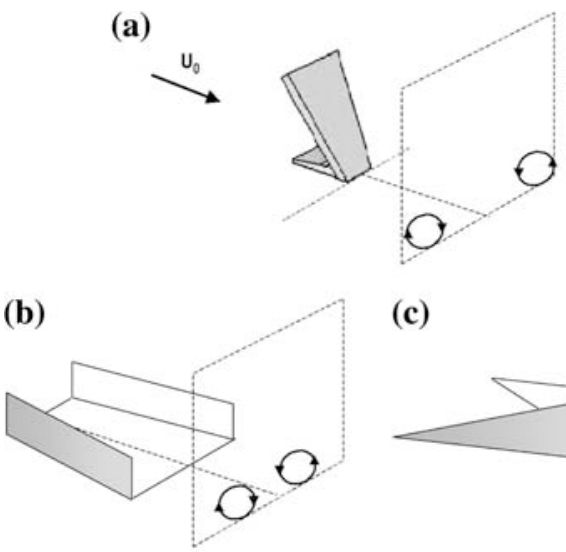

(c)

Fig. 7 Comparison between the vortex generators used in this study (a), and the ones used by Angele and Grewe (2002) (b) or by Betterton et al. (2000) (c) which are classic winglets. A single trapezoidal blade creates a pair of counter-rotating vortices which is usually created by two winglets

\section{http://www.citroen.com/CWW/fr-FR/CONCEPTCARS/ C-AIRLOUNGE/Cairlounge_1/).}

In these passive control experiments, the VGs are set along a line parallel to the $y$ axis (Fig. 6), and are moved downstream along the curved surface. Their position is given in the curvilinear coordinate $s$ as defined on Fig. 2a).

There are many other parameters which will play an important role in the efficiency of the VGs. For a given geometry of the trapezoidal blade, one can still vary four important parameters:

- the angle $\alpha$ between the VG blade and the surface,

- the curvilinear position of the line of VGs on the surface,

- the wavelength $\lambda$ corresponding to the spacing between two neighboring VGs

- the length of the line of VGs, which will depend on the number of VGs used together and of their spacing.

In the following, the influence of the four parameters will be studied. As the space parameter is very large, only a few configurations could be studied for each parameters. Nevertheless, it will give some insights into their influence on the aerodynamic forces.

\section{Parametric study of the influence of the vortex generators on the aerodynamic forces}

\subsection{Influence of the longitudinal position and angle of the vortex generators}

Since it is not possible to predict numerically or through a stability analysis the right perturbation, it is necessary to investigate the receptivity of the flow through a parametric 
study. In this section 17 vortex generators are distributed along the width of the rear slant of the bluff-body, leading to a wavelength $\lambda$ (or spanwise spacing) equal to $0.02 \mathrm{~m}$. Drag and lift measurements are carried out for different curvilinear positions of the VGs on the rear part of the bluff-body. The evolution of the drag and lift as a function of the position of the VGs is shown in Fig. 8 for two different blade angles relative to the wall: $\alpha=60^{\circ}$ (the blade facing the flow as shown on Fig. 6 and $\alpha=120^{\circ}$ (with the tips of the blade pointing downstream). The drag of the bluff-body without flow control for $U_{o}=20 \mathrm{~m} \mathrm{~s}^{-1}$ is $C_{d}=0.32$, while the lift is $C_{l}=0.46$.

The first observation is the strong influence on the drag coefficient: in both cases, the drag is decreased, with a reduction about $-12 \%$. The second observation is the evolution of the drag reduction as a function of the longitudinal position of the VGs: in both cases, one can see a clear single minimum, and a relatively large longitudinal region where the VGs are efficient. The longitudinal extent of these regions is about $0.12 \mathrm{~m}$ long in both cases and the position of the minimum is the same for both configurations: $s_{\min } \simeq 0.20 \mathrm{~m}$ for $\alpha=120^{\circ}$ and $\alpha=60^{\circ}$. The optimal position of the VG line is, in both cases, clearly upstream of the position of the natural separation of the boundary layer $\left(s_{\text {nat }} \simeq 0.26 \mathrm{~m}\right)$. To be noticed is the fact that a significant drag reduction is also obtained when the VGs are located downstream of the natural separation line.

The same kind of evolution is observed for the lift coefficient, with a maximum reduction of more than $-54 \%$ for the $\alpha=60^{\circ}$ case. The optimal position of the VG line is also different in both cases: the maximum lift reduction for $\alpha=120^{\circ}$ is observed $0.02 \mathrm{~m}$ downstream of the $\alpha=60^{\circ}$ configuration.

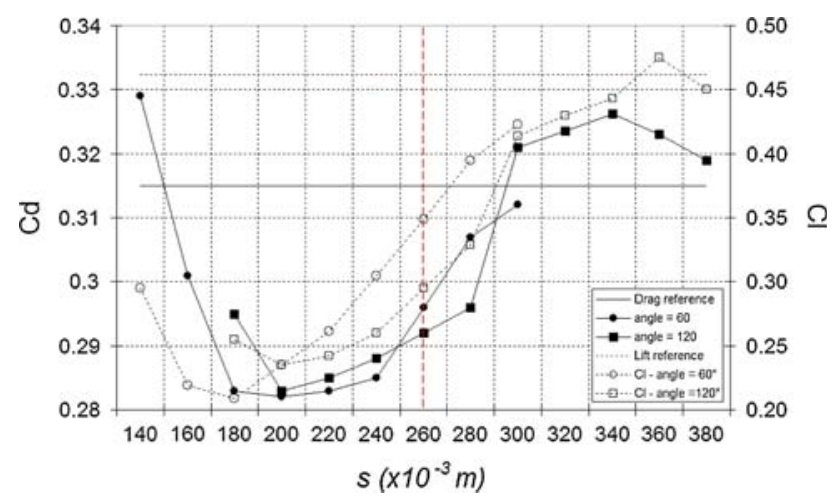

Fig. 8 Influence of the position of the Vortex Generators line on the drag (full lines and left axis) and lift coefficients (dotted lines and right axis) for two angles of the VGs relative to the wall $\left(\alpha=60^{\circ}\right.$ or $\alpha=120^{\circ}$ ) for $U_{o}=20 \mathrm{~m} \mathrm{~s}^{-1}$ and a $0.020 \mathrm{~m}$ spacing. The vertical dotted line indicates the position of the mean natural separation line i.e. without vortex generators. The horizontal lines indicate the drag and lift reference values
The rear-axle lift follows the same evolution as the total lift, with a minimum located on the same curvilinear position. The reduction is much higher than the global lift: $-93.3 \%$ for the $\alpha=60^{\circ}$ configuration and $-90.7 \%$ for the $\alpha=120^{\circ}$ case. The difference with the global lift is easily explained as the vortex generators modify the pressure distribution over the rear slant of the model. This result is of course very interesting from the automotive engineer point of view as the rear-axle lift is very important for the stability of a vehicle.

\subsection{Influence of the spacing between the vortex generators}

It is well known from previous studies (Godard and Stanislas 2006; Lin et al. 2002) that the spacing between the $3 \mathrm{D}$ perturbations induced by vortex generators has a strong influence on their efficiency on the forced flow. The optimal amplification of the perturbations by the boundary layer is closely associated to its stability properties. When the objective is to control a separated flow, one has to deal with two challenges: the first is to find the right perturbations that will be amplified by the boundary layer (Duriez et al. 2009; Andersson et al. 2002), while the second is to modify the properties of the boundary layer in the proper way to control the separated flow (Duriez et al. 2008). These are still open questions and very active research fields. Parametric studies are still the only way to search for critical spacing leading to better results for a given configuration of VGs.

In this section, the spacing between the VGs is changed from $\lambda=0.02 \mathrm{~m}$ to $\lambda=0.015 \mathrm{~m}$. As the VGs are distributed along the all width of the rear slant, the number of VGs is also changed from 17 to 22 . The same kind of drag and lift evolution as in the previous section is plotted on Fig. 9. One can notice that the drag and lift longitudinal evolutions are clearly different from the ones obtained with the $0.020 \mathrm{~m}$ spacing. The comparison between the two spacings is easier if substracting the drag (or lift) coefficients obtained with a $0.015 \mathrm{~m}$ spacing to the one obtained with a $0.020 \mathrm{~m}$ spacing (Fig. 10). The first observation is that the drag or lift reductions are larger with a $0.015 \mathrm{~m}$ spacing for nearly all the position and for the two different angles. The most important difference is the longitudinal extent of the region where the drag is reduced. One can see a drag reduction with a line of VGs up to $s \approx 0.32 \mathrm{~m}$, i.e. further downstream of the previous case, and even downstream of the natural separation line. One can also notice that when the VGs are located at $s=0.14 \mathrm{~m}$, the drag is strongly increased reaching a very high value $\left(C_{d}=0.405\right)$. This drag value has not been included in the graph to make the downstream evolution of the drag clearer especially in the region where the drag is minimum. 


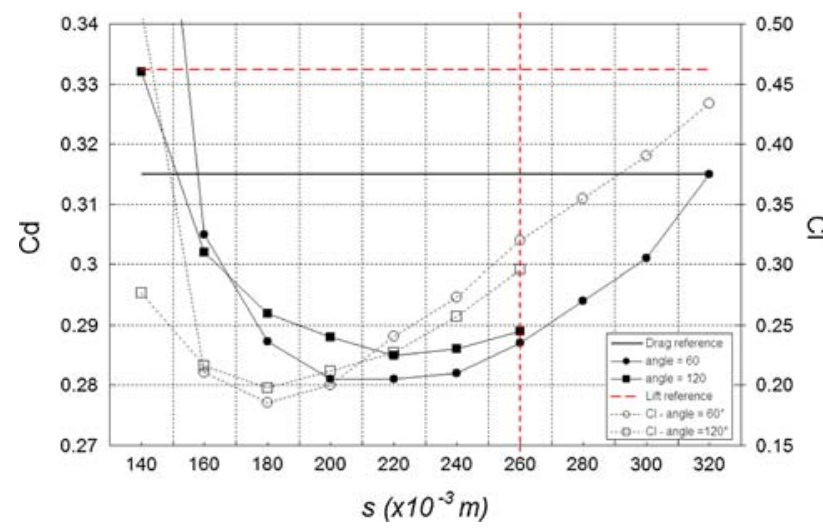

Fig. 9 Influence of the position of the Vortex Generators line on the drag (full lines and left axis) and lift coefficients (dotted lines and right axis) for two angles of the VGs relative to the wall $\left(\alpha=60^{\circ}\right.$ or $\alpha=120^{\circ}$ ) for $U_{o}=20 \mathrm{~m} \mathrm{~s}^{-1}$ and a $0.015 \mathrm{~m}$ spacing. The vertical dotted line indicates the position of the mean separation line. The horizontal lines indicate the drag and lift reference values

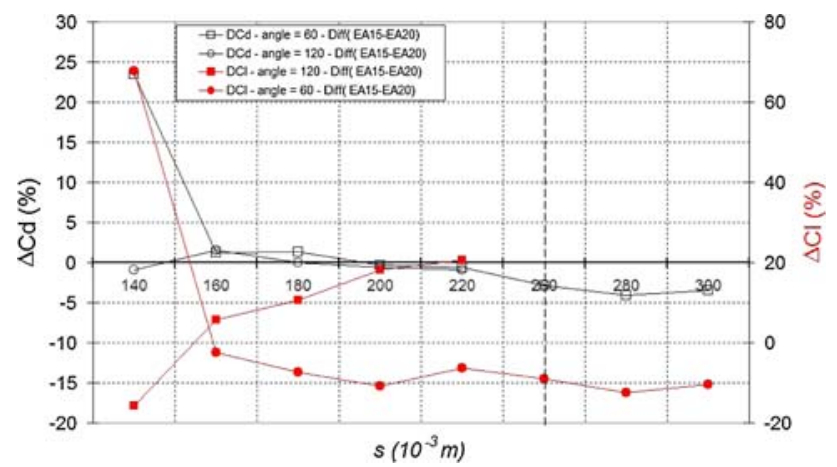

Fig. 10 Difference between the drag (or lift) of the bluff-body obtained with a $0.015 \mathrm{~m}$ spacing and the a $0.020 \mathrm{~m}$ spacing for $U_{o}=20 \mathrm{~m} \mathrm{~s}^{-1}$ and for $\alpha=120^{\circ}$ and $\alpha=60^{\circ}$. Open (respectively full and red) markers are used for the drag (respectively lift) coefficient. One can see that the a $0.015 \mathrm{~m}$ spacing leads to better results in most of the cases

The optimal longitudinal position for the lift reduction is different than the one for the drag reduction in the $\alpha=120^{\circ}$ case. We also emphasize the fact that we can still observe a lift reduction, in the case $\alpha=120^{\circ}$, when the line of VG is located downstream of the natural separation line. From a practical point of view, the $\alpha=60^{\circ}$ may be more interesting, as the drag and lift reduction is optimized for nearly the same longitudinal position of the VGs line. The lift reduction is also slightly larger $(-61 \%)$ than in the $\lambda=15 \mathrm{~mm}$ configuration.

As in the previous section, the rear-axle lift follows the same evolution as the total lift, with a minimum located on the same curvilinear position. The reduction is also much higher than the global lift: $-103.9 \%$ for the $\alpha=60^{\circ}$ configuration and $-101 \%$ for the $\alpha=120^{\circ}$ case. In both (a)

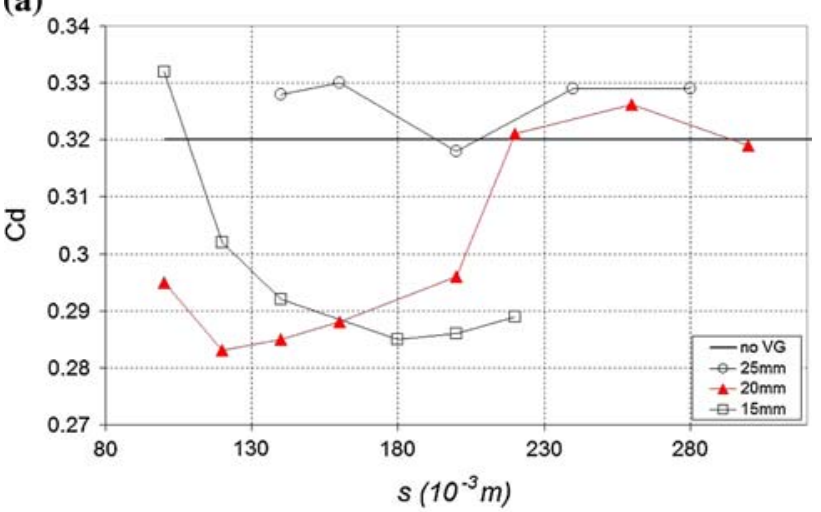

(b)

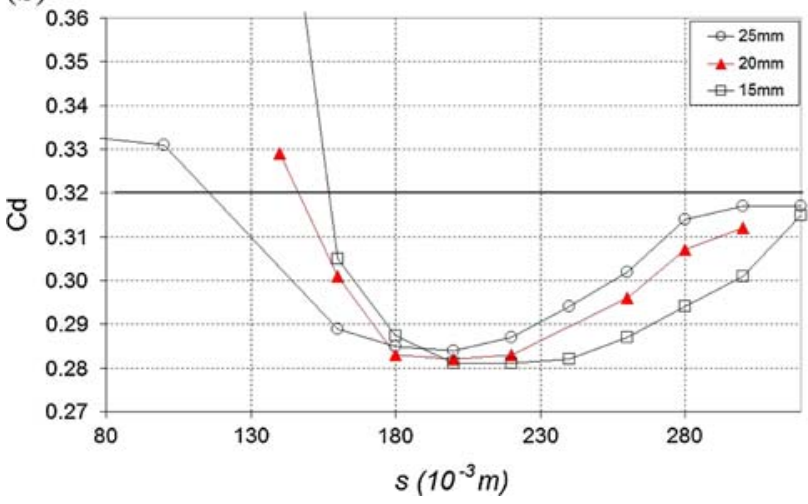

Fig. 11 Influence of the spacing between the vortex generators on the drag of the bluff-body $\left(U_{o}=20 \mathrm{~m} \mathrm{~s}^{-1}\right)$ and for $\alpha=120^{\circ}$ (a) and $\alpha=60^{\circ}(\mathbf{b})$

cases, the sign of the lift on the rear axle is reversed, i.e. the model is no longer lifted up but pulled down to the ground. Such a result is of course very interesting for automotive applications to improve longitudinal stability of the vehicle. The slight differences between the optimal positions for drag or lift reduction and the much larger lift reduction compared to the drag reduction can be explained by the fact that the lift force is mainly associated to the pressure distribution over the rear slant. The low pressure over the rear slant is at the origin of the vertical force pulling upward the vehicle. The origin of the drag force is more complex and is also influenced by the longitudinal structure as will be discussed in the last section. The large lift reduction shows that the wall pressure distribution is strongly modified, and the pressure globally increased over the rear slant by the VG.

In Fig. 11a, the drag evolution is plotted as a function of the curvilinear position of the vortex line for three different spacings for the VGs $(\lambda=15,20$ and $25 \mathrm{~mm})$ and for the $\alpha=120^{\circ}$ configuration. As mentioned previously, the VGs are regularly distributed along the width of the model so that the $\lambda=25 \mathrm{~mm}$ configuration is obtained with $13 \mathrm{VGs}$. 
The same kind of longitudinal evolution is recovered, but a strong influence of the spacing of the VGs on the efficiency of the vortex line can be noticed: if the spacing is too large the line of VGs looses its efficiency. It seems reasonable to assume that there exist an optimal spacing (in this case, around $20 \mathrm{~mm}$ ) to get the maximum drag and/or lift reduction.

If the same experiment is carried out with the $\alpha=60^{\circ}$ configuration, the influence of the spacing is very different. As a matter of fact, this configuration appears to be far less sensitive to the wavelength (Fig. 11b). The optimal position as well as the maximum drag reduction are nearly the same for the three wavelengths. The only clear difference is the extent of the longitudinal regions where the line of VGs is efficient. As in the previous section, the $\alpha=60^{\circ}$ configuration is interesting because of its dependence on the spacing of the VGs. The relatively large differences between the drag evolution shown on Fig. 11a and $\mathrm{b}$ can be interpreted as a clear indication that the perturbations induced by the VGs are also different when $\alpha=60^{\circ}$ or $\alpha=120^{\circ}$. The $\alpha=120^{\circ}$ configuration is much more sensitive to a spacing variation than the $\alpha=60^{\circ}$ configuration suggesting that the longitudinal vortices induced by a single VG $(\lambda=\infty)$ are much weaker in the case $\alpha=120^{\circ}$ than in the case $\alpha=60^{\circ}$. This would require a detailed analysis of the flow over isolated VGs with different angle $\alpha$ to confirm such an hypothesis.

The right curvilinear position for the VGs is approximately $50-60 \mathrm{~mm}$ upstream of the location of the separation line. Nevertheless, there is still a drag reduction when the line of VGs is located downstream of the natural separation line. This is also an interesting result, even if it cannot be explained for the moment as it would require a detailed investigation of the near wake for this configuration. Nevertheless, two hypothesis can be drawn. The first is that the VGs do not modify the recirculation bubble. In this case, most of the VGs are into the recirculation bubble, but the VGs close to the side edges can perturb the longitudinal trailing vortices leading to a slight drag reduction. The second hypothesis is that the perturbation induced by the VGs is strong enough to modify the boundary layer upstream of the VGs.

Before turning to next section, one should mention here that a classic rear spoiler has also been tested on the model. It was an important question we had to answer indeed: are the VGs more or less efficient than a rear spoiler, which is the classic device used in automotive aerodynamics? To answer this question, a spoiler has been tested on the same curvilinear position where the VGs are the most efficient, i.e. at $s=0.2 \mathrm{~m}$. It leads to a $6.3 \%$ drag reduction, for a total $62.8 \%$ lift reduction corresponding to a $107.3 \%$ reduction of the rear-axle lift. The rear spoiler gives a comparable lift reduction but a lower drag reduction than the best configurations of vortex generators.

\subsection{Influence of the Reynolds number}

After exploring the sensitivity of the aerodynamic forces to both wavelength and curvilinear position of the perturbations induced by the VGs for a given free-stream velocity, the influence of the Reynolds dependence still has to be investigated (Table 1). The objective here is to check if the VGs remain efficient even for higher Reynolds number.

The influence on the drag coefficient of a given line of VGs $\left(\alpha=60^{\circ}\right.$ and $\left.\lambda=0.015 \mathrm{~m}\right)$ as a function of the longitudinal position of the line is investigated for three free-stream velocities. The result is shown in Fig. 12. One can notice that the drag reduction (compared to the respective reference values) obtained with a given configuration is decreasing: $-12.2 \%$ for $U_{0}=20 \mathrm{~m} \mathrm{~s}^{-1},-7.1 \%$ for $U_{0}=30 \mathrm{~m} \mathrm{~s}^{-1}$ and $-3.7 \%$ for $U_{0}=40 \mathrm{~m} \mathrm{~s}^{-1}$ (every gains are relative to the drag reference value obtained for each freestream velocity).

The same configuration with a different angle $\left(\alpha=40^{\circ}\right)$ is studied for $U_{o}=40 \mathrm{~m} \mathrm{~s}^{-1}$ and $s \simeq 0.2 \mathrm{~m}$. Thanks to this small modification, one can get a $-7.1 \%$ drag reduction. To keep a significant drag or lift reduction, even if the freestream velocity is changing, it will be necessary to use Active Vortex Generators. It implies to find a proper dynamic parameter to be used in a closed-loop experiment. The previous results with passive actuators clearly show that the angle of the blade compared to the wall could be driven as a function of the freestream velocity or other parameters like wall pressure in critical area. It will be very useful for automotive aerodynamics to deal with many transients situations like wind gusts, vehicles crossings or different speed limits.

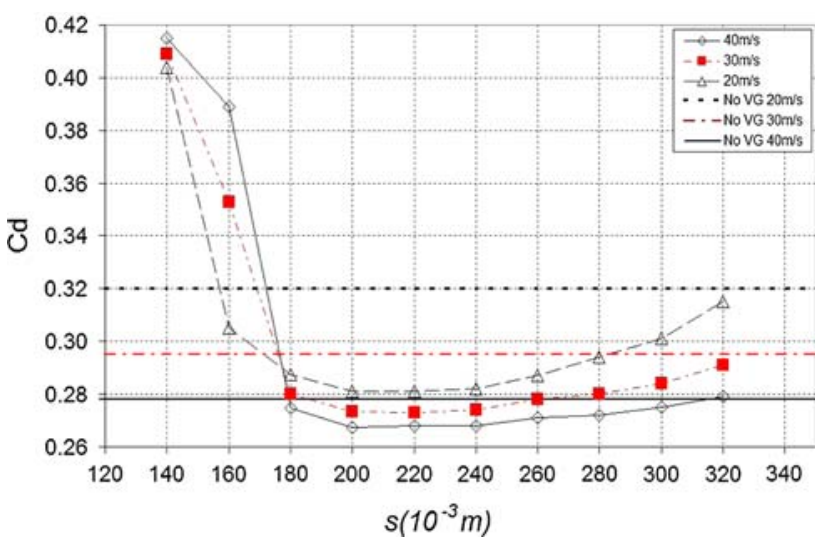

Fig. 12 Drag reduction obtained with a line of a given VG $\left(\alpha=60^{\circ}\right.$ and $\lambda=0.015 \mathrm{~m}$ ) for three different free-stream velocities for $U_{o}=20,30$ and $40 \mathrm{~m} \mathrm{~s}^{-1}$ 
Fig. 13 Sketch of the three configurations studied, with three distributions of the VGs along the width of the model for $s=0.20 \mathrm{~m}$

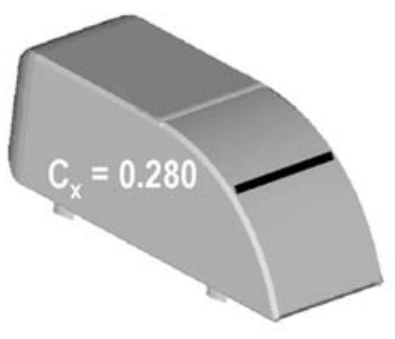

Complete line

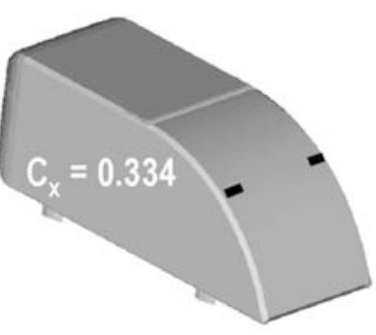

4 VG on each side

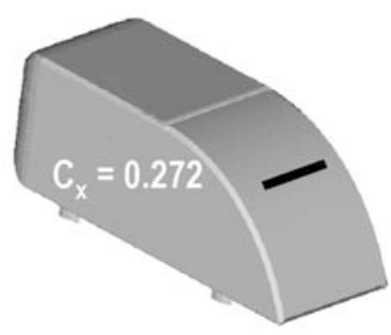

14 VG in the center
Table 2 Drag coefficients for different distributions of VGs along the line $s=0.20 \mathrm{~m}$

\begin{tabular}{lllll}
\hline & $\begin{array}{l}\text { Reference } \\
\text { flow }\end{array}$ & $\begin{array}{l}\text { Complete } \\
\text { line }\end{array}$ & $\begin{array}{l}14 \text { VGs in } \\
\text { the center }\end{array}$ & $\begin{array}{l}4 \text { VGs on } \\
\text { each side }\end{array}$ \\
\hline$C_{d}$ & 0.315 & 0.280 & 0.272 & 0.334 \\
\hline
\end{tabular}

\subsection{Influence of the distribution of the vortex} generators along the width of the model

The following step in the analysis of the drag reduction mechanism is to identify the VGs which are responsible for the diminution of the trailing vortices intensity. Only one configuration is considered for this study: 22 VGs distributed along the width of the model with a $\lambda=0.015 \mathrm{~m}$ spacing and a $60^{\circ}$ angle. To evaluate the importance of the different regions of the line of VGs, a few VGs have been removed from the center or from the sides of the optimal line of VGs ( $s=0.20 \mathrm{~m}$ on Fig. 9), as shown on Fig. 13 . The row of VGs is still located at $s=0.20 \mathrm{~m}$ where the drag is minimum for this configuration, and some of the 22 VGs homogeneously distributed along the width are removed. Two new configurations are then studied with the same spacing $(\lambda=0.015 \mathrm{~m})$ :

- 4 VGs located on each side edge of the rear part of the model are removed

- 14 VGs located in the center of the line of VGs are removed

Table 2 reports values of the drag coefficient for the different configurations described in Fig. 13. It shows that when the VGs are only located on each side of the model the drag coefficient is even higher than the reference drag coefficient. This result suggests that the VGs close to the side edges of the rear slant interact strongly with the trailing vortices. As the recirculation bubble is probably not modified, one can think that the VGs make the trailing vortices stronger, leading to an increase in the drag coefficient. On the contrary, when the VGs located close to the side edges are removed, the remaining VGs have an even more favorable effect on the drag coefficient than the complete line. This result is coherent with the previous one: the VGs in the center clearly modify the recirculation bubble leading to a drag reduction, while the VGs close to the side edge seems to make the trailing vortices stronger leading to a drag increase. As a consequence, removing the VGs close to the side edges leads to a better drag reduction. This result will be confirmed in the following when investigating the velocity field in the near-wake of the bluff-body.

\subsection{Toward active mechanical vortex generators}

The previous results demonstrate the dependance of the drag or lift to the different parameters. It allows the identification of a good dynamical parameter: the angle $\alpha$ between the blade and the wall. In a perspective of closedloop experiment and of the search of a more efficient way to find the optimal parameters, it is important to propose a new set-up where the dynamical parameter can be remotely driven. In this section, we present an experiment performed using a motorized model. The vortex generators are now attached to a rotating axis (Fig. 14b) allowing the exploration of every angles between $0^{\circ}$ to $90^{\circ}$ without having to stop the wind tunnel. The rotation of the axis is remotecontrolled from outside of the wind tunnel, so that the wind blowing is stopped only to modify the longitudinal position of the VGs.

The experiments has been carried out with slightly different geometric parameters than the ones used in the previous sections: the VGs are now $15 \mathrm{~mm}$ long, the spacing is $12 \mathrm{~mm}$ and only twelve VGs are used instead of fourteen in the previous section. The reason is that we found from preliminary investigations that this configuration is slightly better than the one studied in the previous section. The model has been modified to integrate the motors and mechanics necessary to pilot the rotation of the VG, but the overall dimensions and geometry are strictly the same, so that the results are comparable (Fig. 14a).

Both drag and lift coefficients are measured for the three freestream velocities and for thirteen curvilinear position $s$ (200 $\mathrm{mm}<s<300 \mathrm{~mm}$ ) of the line of VGs over the rear part of the model. For each curvilinear position, drag and lift are measured every ten degrees, for $\alpha$ ranging between 
Fig. 14 Picture of the rear of the model (a) equipped with a set of VGs attached on a motorized rotating axis $(\mathbf{b})$. The rotation of the axis is driven through a remote radio-control system located outside the wind-channel

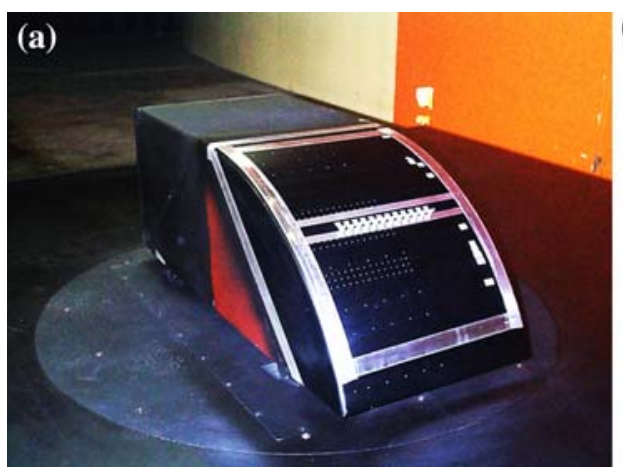

(b)

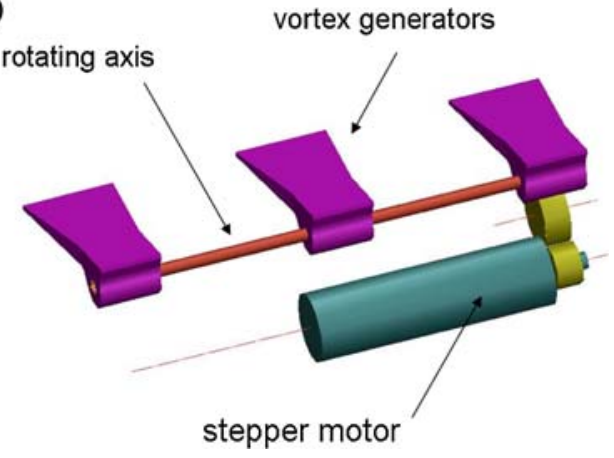

$0^{\circ}$ and $90^{\circ}$. The following results represent as much as 390 different drag (and lift) measurements for each freestream velocity.

In Table 3, the maximum drag and lift reduction obtained with these configurations are summarized. The drag or lift reduction decrease when the Reynolds number is increased, but the results show more than $14 \%$ drag reduction for $U_{o}=20 \mathrm{~m} \mathrm{~s}^{-1}$ (better than the previous studies) and more than $10 \%$ drag reduction for $U_{o}=40 \mathrm{~m}$ $\mathrm{s}^{-1}$. This is another illustration of the importance of a good parametric study and of the sensitivity to both curvilinear position and angle of the VG. The results obtained with a classic rear spoiler located at $s=0.2 \mathrm{~m}$ are also included in the table. The rear spoiler is $0.01 \mathrm{~m}$ long (as long as the $\mathrm{VG})$ with an angle $\alpha=60^{\circ}$ relative to the wall. One can see that the VGs give better results for the drag, while the rear spoiler gives better results for the lift.

These results are more interesting when plotted as contours of drag and lift modification (in percentage compared to the reference case) as a function of the longitudinal position versus the angle $\alpha$ for the three freestream velocities (Fig. 15). This compact representation allows for a simple identification of the optimal parameters: for each velocity, one can identify the minimum of drag or lift in the space parameter $(s, \alpha)$, i.e. the optimal parameters for the given free-stream velocity. The left column corresponds to the contours of drag modification for increasing velocity from top (a) to bottom (e), while

Table 3 Maximum drag and lift reduction for the three freestream velocities. One can also notice the strong reduction of the rear axle lift. For the sake of comparison with a common aerodynamic device, a rear spoiler has also been tested. The results are given in the parenthesis next to reductions obtained with the VGs

\begin{tabular}{llll}
\hline $\begin{array}{l}U_{o} \\
\left(\mathrm{~m} \mathrm{~s}^{-1}\right)\end{array}$ & $\begin{array}{l}\Delta C_{d}(\%) \mathrm{VGs} \\
\text { (Rear spoiler) }\end{array}$ & $\begin{array}{l}\Delta C_{l}(\%) \mathrm{VGs} \\
(\text { Rear spoiler) }\end{array}$ & $\begin{array}{l}\Delta C_{l \text { Rear }}(\%) \mathrm{VGs} \\
\text { (Rear spoiler) }\end{array}$ \\
\hline 20 & $-14(-6.3)$ & $-44.4(-62.8)$ & $-89.4(-107.3)$ \\
30 & $-11.3(-0.3)$ & $-38.4(-57.8)$ & $-92.2(-107.5)$ \\
40 & $-10.6(+2.88)$ & $-39.0(-52.8)$ & $-99.2(-109)$ \\
\hline
\end{tabular}

the right column corresponds to the contours of lift modification.

The first observation is the strong dependency on both the location of the VGs and the angle. For both drag and lift and for all the Reynolds number one can see a sharp frontier (the yellow lines) between the regions of the space parameter where the drag is increased or decreased. For $0.20 \mathrm{~m}<s<0.24 \mathrm{~m}$ there is a critical angle above which the VGs increase the drag or lift forces. One can also notice that for each Reynolds number, the optimal parameters $(s, \alpha)$ are slightly different for drag and lift. For a given position of the VGs, it will be possible to modify the angle to optimize either the drag or the lift.

The Reynolds dependency of both drag and lift is also clear. In both cases, there is a decrease of the spatial extent of the region where the VGs are efficient (green-blue regions on the contour fields), while the unfavorable region is clearly increased. It appears that the curvilinear position $s=0.265 \mathrm{~m}$ is a good compromise to keep nearly the best drag reduction for all Reynolds number, if the angle is well controlled. The problem is that this position will not be the best for the lift coefficient. As a matter of fact, $s=0.25 \mathrm{~m}$ would be better for an optimal lift reduction. It confirms that it will be difficult to optimize both drag and lift with a given configurations. Nevertheless, once the optimal location of the VGs is chosen to minimize one of the forces, the control of the angle will allow the reduction of the other force.

It is also interesting to notice that the region in the space parameter $(s, \alpha)$ where the lift is reduced is larger than the one where the drag is reduced. The lift reduction is also larger. From this point of view, the location of the VGs should then rather be chosen to optimize the drag. Of course the contours presented here will be different if the spacing or the height of the VGs are changed. Here, one should mention that similar experiments with a motorized device were carried out on a full-scale vehicle, demonstrating that the drag and lift reduction are comparable with a slightly stronger dependency on the angle than on the bluff-body (Despré et al. 2003; Aider et al. 2009b). 

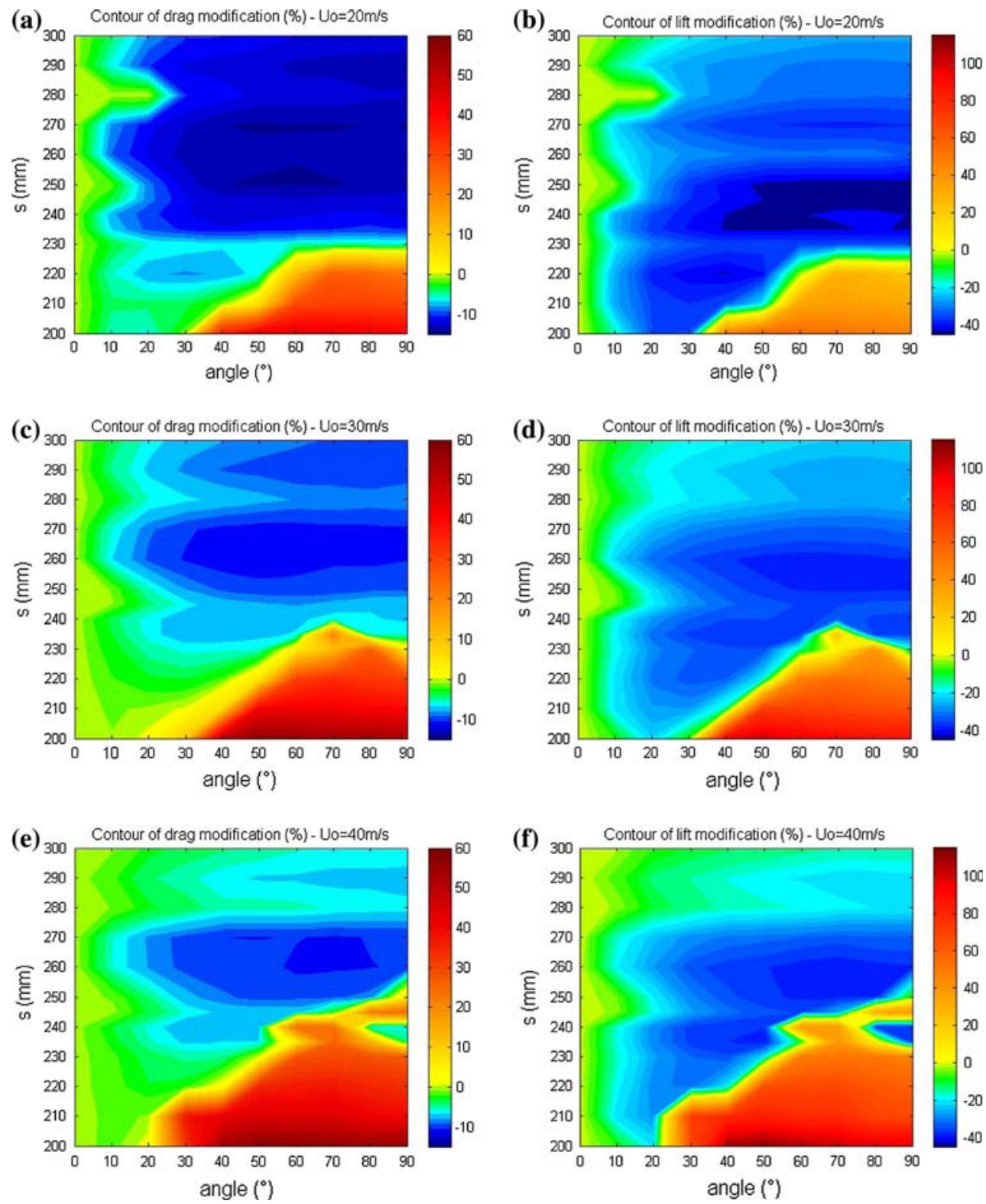

Fig. 15 Contour plots of drag and lift reduction obtained for three freestream velocities: $U_{0}=20 \mathrm{~m} \mathrm{~s}^{-1}(\mathbf{a}, \mathbf{b}), U_{0}=30 \mathrm{~m} \mathrm{~s}^{-1}(\mathbf{c}, \mathbf{d})$ and $U_{0}=40 \mathrm{~m} \mathrm{~s}^{-1}(\mathbf{e}, \mathbf{f})$. Drag reduction are shown on the left column and the lift reduction on the right one. Each contour plot gives

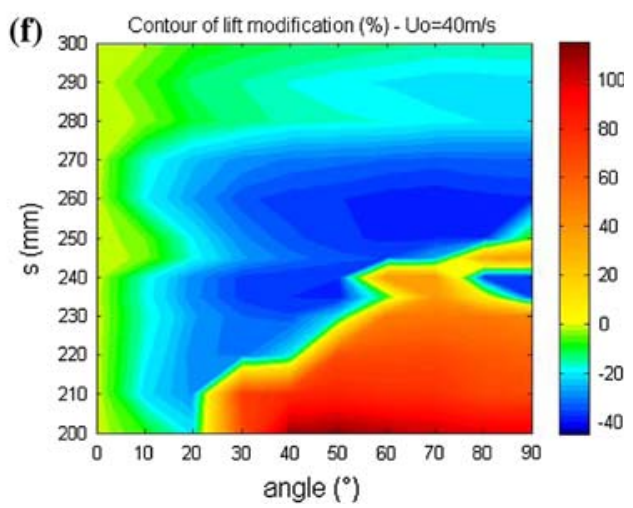

a global view of the influence of both the position of the line of vortex generators and of the angle of the blades compared to the wall. It represents as much as 390 drag and lift measurements

\section{Investigation of the near-wake of the bluff-body}

\subsection{Boundary layer over the bluff-body}

The previous sections illustrate the high dependance of the aerodynamic forces on the VGs and also the difficulty in understanding the way the VGs interact with the boundary layer. If some global quantities characterizing the modification of the base flow can be estimated a priori to choose the right position of the VGs upstream a separated flow

over a rounded wall (Duriez et al. 2006, 2008), it still is necessary to lead extensive parametric studies to choose the right parameters in the case of 3D bluff-bodies. It is also necessary to have a better insight into the way the VGs modify the near-wake of the bluff-body through PIV and hot-wire measurements.

First, hot-wire velocimetry was used to measure the longitudinal evolution of the boundary layer over the bluffbody without vortex generators (Fig. 16). The boundary layer thickness is calculated using the $0.99 \% U_{o}$ definition 
for the boundary measured on the top of the model. Over the rounded rear slant, the boundary layer is accelerated and the boundary layer thickness is measured where the velocity is maximum. The boundary layer upstream of the position of the VG line $(s=-0.102 \mathrm{~m})$, is already $1.7 \times 10^{-2} \mathrm{~m}$ thick. At the position where the VG line begins to reduce the drag $(s=0.150 \mathrm{~m})$, the boundary layer is already $3 \times 10^{-2} \mathrm{~m}$ thick, before reaching $3.5 \times 10^{-2} \mathrm{~m}$ where the drag reduction is maximum. This is to be compared to the effective height of the VG seen by the flow i.e. $h_{e}=h \cdot \sin (\alpha)=8.6 \times 10^{-3} \mathrm{~m}$ which is smaller than the boundary layer thickness: $h_{e} / \delta<0.5$ so that the VGs work like sub-boundary or low-profile vortex generators as defined by Lin (2002).

\subsection{Investigation of the velocity field in the near wake of the bluff-body}

In order to understand the modification of the base flow by the VGs, wall pressure for different $s$ values and velocity field measurements in different planes in the near-wake were carried out. In addition to the reference flow, only two significantly different cases are discussed, namely a high drag case $\left(C_{d}=0.405\right.$ with the VGs located at $\left.s=0.14 \mathrm{~m}\right)$ and the low drag case $\left(C_{d}=0.280\right.$ with the VGs located at $s=0.22 \mathrm{~m})$ obtained with $22 \mathrm{VGs}\left(\lambda=15 \times 10^{-3} \mathrm{~m}\right)$ distributed along the width of model and with the $\alpha=60^{\circ}$ configuration (Fig. 9).

Mean PIV and wall pressure measurements are both displayed in Fig. 17 for the low drag configuration (Fig. 17e and f) and the high drag configuration (Fig. 17c and d). It clearly shows that the separation of the boundary layer over the rear slant occurs just upstream the VGs suggesting that the VGs do no not

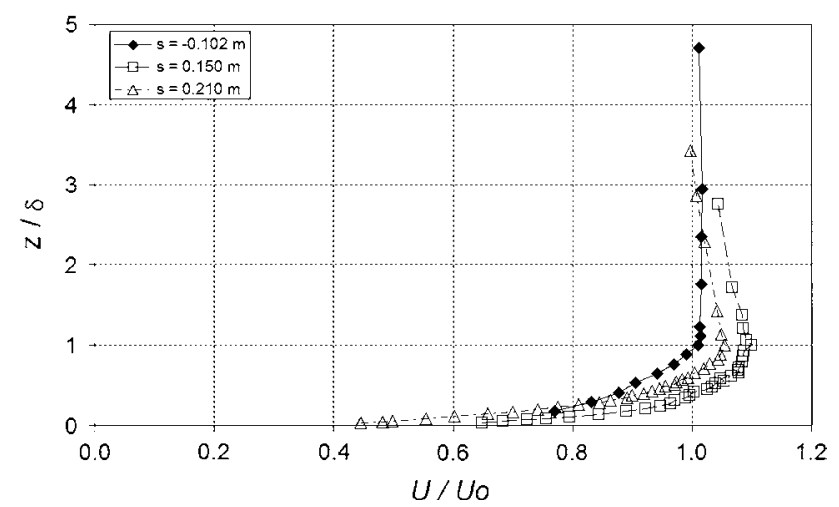

Fig. 16 Evolution of the boundary layer along the curved rear part of the model without Vortex Generators for $U_{o}=20 \mathrm{~m} \mathrm{~s}^{-1}$. The boundary layer grows from $\delta \approx 1.7 \times 10^{-2} \mathrm{~m}$ on the top of the model $(s=-0.102 \mathrm{~m})$ to $\delta \approx 3.5 \times 10^{-2} \mathrm{~m}$ on the rounded rear slant upstream the separation $(s=-0.210 \mathrm{~m})$ and in the region where the drag reduction is the highest delay but rather trigger the separation of the boundary layer. This behavior is not common and was not expected, as most of the time the vortex generators are used to delay the separation in $2 \mathrm{D}$ configuration like around an airfoil. To explain such a behavior, a detailed investigation of the flow around the vortex generators is needed which is beyond the scope of this study. Nevertheless, one can think that the configuration triggering the separation of the boundary layer induces a deceleration of the boundary layer. A tentative explanation is the following. It is well known that the vortex generators create counter-rotating streamwise vortices which are responsible for a mixing of the boundary layer through two types of regions (Fig. 18):

- inflow regions, which are the regions where the highmomentum fluid from above the boundary layer is taken toward the wall,

- outflow regions, which are the regions where the lowmomentum fluid from the boundary layer is taken outside the boundary layer.

In a turbulent boundary layer, the non-linearities breakdown the balance between inflow and outflow regions. Depending on the vortex generators, the boundary layer can be either dominated by the inflow regions and globally accelerated (and then stronger against an adverse pressure gradient) or, on the contrary, dominated by the outflow region and globally decelerated (Duriez et al. 2008). The triggering of separation of the boundary layer can then be associated to a boundary layer dominated by the outflow regions.

The separation line is located just upstream from the VGs where a relative pressure increase is measured. In the recirculation bubble, downstream from the VGs, the pressure coefficient is nearly uniform and reaches significantly different mean values in the high or low drag case (respectively -0.40 and -0.15 ), which is consistent with the drag and lift increase (respectively decrease). Besides, the separation region is much larger in the low drag situation (Fig. 17e).

Figures 19 and 20, respectively represent the mean flow velocity magnitude and the corresponding streamwise vorticity $\omega_{x}$ (which is only weakly altered by the streamwise velocity component) in the plane $x=0.58 \mathrm{~m}$ (i.e. $0.13 \mathrm{~m}$ downstream from the model as shown on Fig. 19a) in the three considered cases. The PIV field is measured on one half of the near-wake, assuming symmetry for the mean velocity field. The bluff-body is shown behind the PIV field to help the reading. One can see the footprint of a longitudinal counter-rotating vortex crossing this plane, corresponding to the two trailing vortices mentioned in Fig. 1b. Considering both Figs. 19 and 20 reveals that the 
Fig. 17 Modification of the near-wake of the bluff-body by the VGs. Left column $(\mathbf{a}, \mathbf{c}, \mathbf{e})$ wall pressure $\left(C_{p}\right)$ measurements coupled with the mean flow velocity in the plane $y=0$; the black circles represent the pressure taps location. Streamlines have been added in the symmetry plane to help the visualization of the recirculation bubble. Right column (b, d, f) mean flow velocity in the planes $y=0.12 \mathrm{~m}$ and $z=0.15 \mathrm{~m}$ crossing the longitudinal vortices; the planes $y=\mathrm{cst}$ and $z=$ cst are respectively colored by $\sqrt{u^{2}+w^{2}}$ and $\sqrt{u^{2}+v^{2}}$. First row $(\mathbf{a}, \mathbf{b})$ reference flow $\left(C_{d}=0.315\right)$. Second row $(\mathbf{c}, \mathbf{d})$ high drag case $(s=0.14 \mathrm{~m}$ and $\left.C_{d}=0.405\right)$. Third row $(\mathbf{e}, \mathbf{f})$ low drag case ( $s=0.20 \mathrm{~m}$ and $C_{d}=0.280$ ). The black thick line shows the location of the VGs. The thin line indicates the location of the natural separation $s_{\text {nat }}$
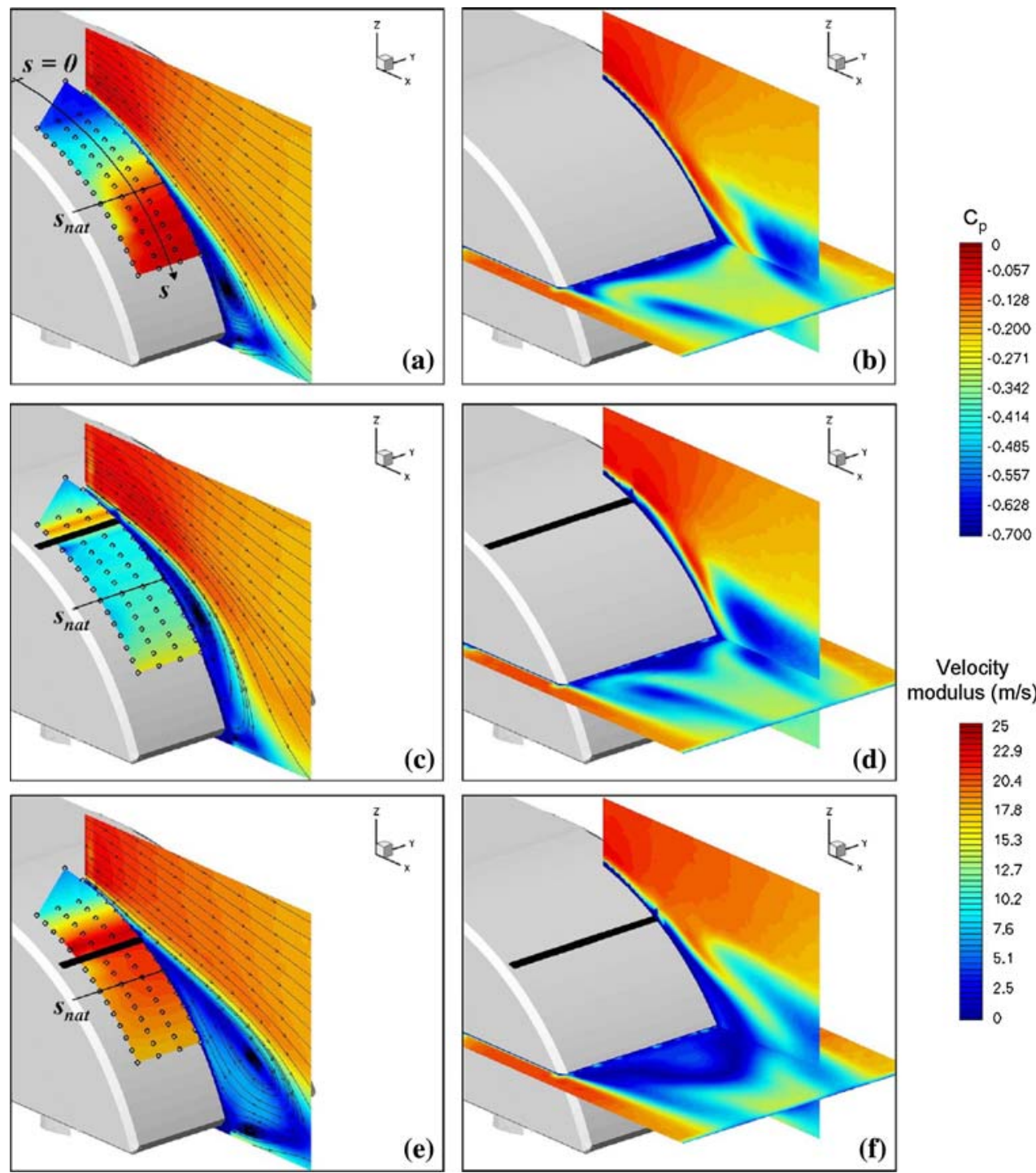

Velocity modulus $(\mathrm{m} / \mathrm{s})$

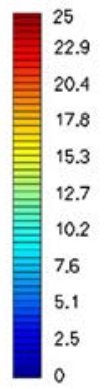

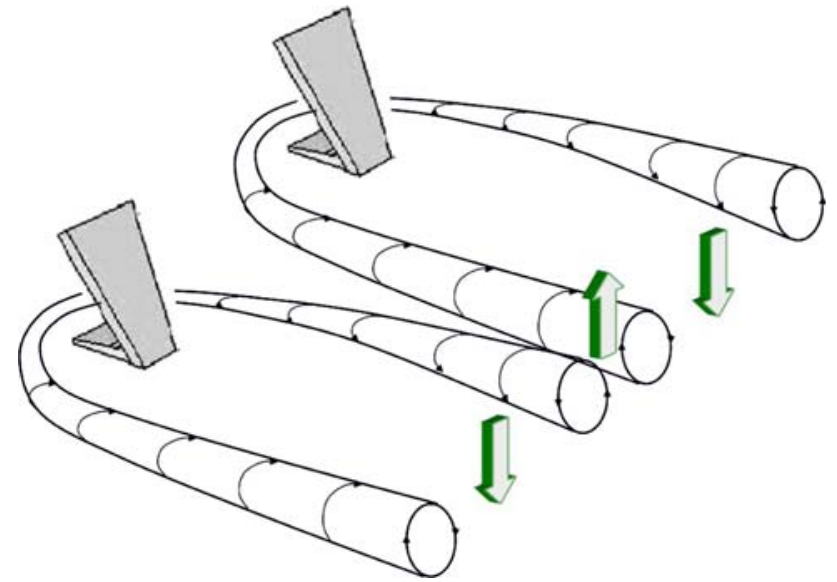

Fig. 18 Schematic representation of the inflow (arrows pointing downwards) and outflow regions (arrows pointing upwards) created between the streamwise counter-rotating vortices induced by the vortex generators
VGs have also a significant effect on the longitudinal structures. In particular, the downwash between the vortices is strongly dependent on the position of the VGs, suggesting that its contribution to the global drag could have changed. For $s=0.14 \mathrm{~m}$ it is higher $\left(C_{d}=0.405\right)$ than in the natural case $\left(C_{d}=0.315\right)$, whereas it is lower for $s=0.20 \mathrm{~m}\left(C_{d}=0.280\right)$. Figure 20 shows that, on the one hand, the highest vorticity is obtained in the reference case and the lowest in the low drag case; but on the other hand, the high vorticity region is the largest in the high drag case.

The streamwise vortices strength can be evaluated by the circulation $\Gamma$ along a properly chosen contour. Thus, we define $\mathcal{C}$ as the contour corresponding to the surface $\mathcal{S}$ characterized by the following conditions: $0 \leq y \leq 0.15 \mathrm{~m}$ and $0 \leq z \leq 0.30 \mathrm{~m}$ (here, the symmetry of the timeaveraged flow is assumed so that the two counter-rotating 
Fig. 19 PIV measurements in the vertical plane $x=0.58 \mathrm{~m}$ (i.e. $0.13 \mathrm{~m}$ downstream from the model) colored by $\sqrt{v^{2}+w^{2}}$. a location of the PIV plane. $\mathbf{b}$ reference flow $\left(C_{d}=0.315\right)$. $\mathbf{c}$ high drag case $\left(s=0.14 \mathrm{~m}\right.$ and $\left.C_{d}=0.405\right)$. d low drag case $(s=0.22 \mathrm{~m}$ and $C_{d}=0.280$ ) (a)

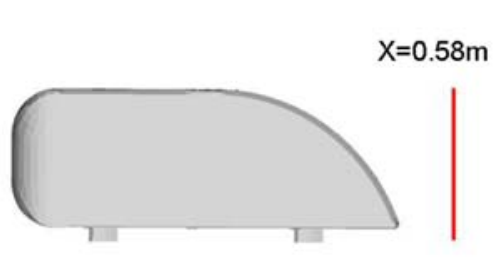

(c)

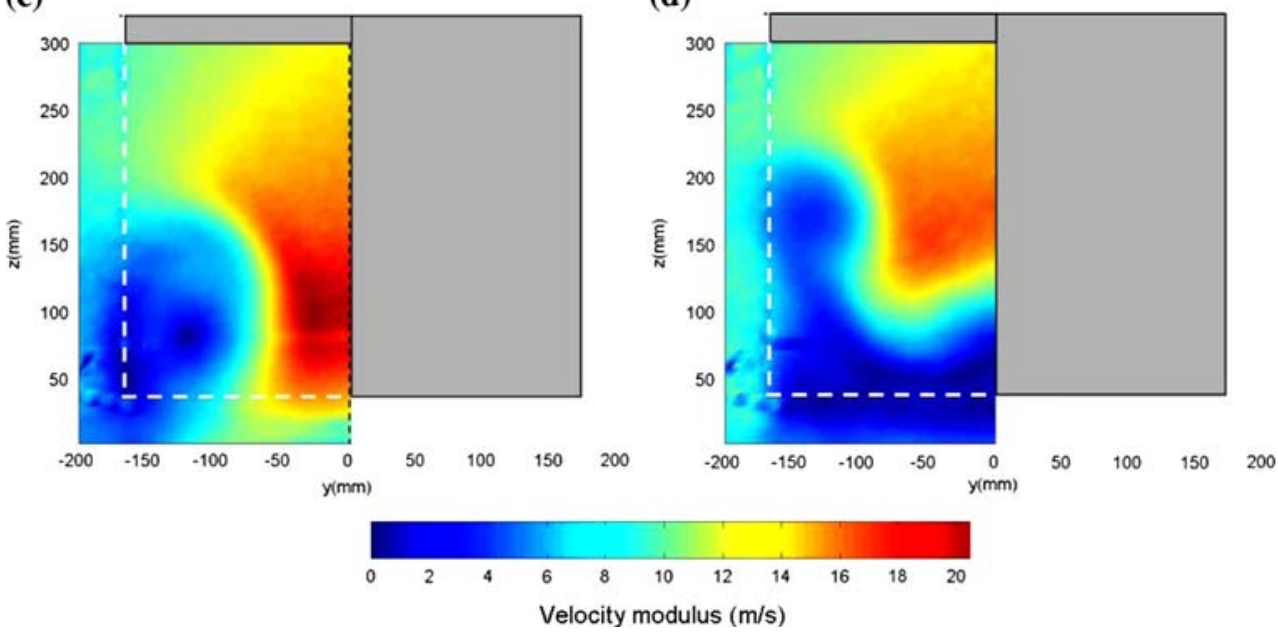

(b)

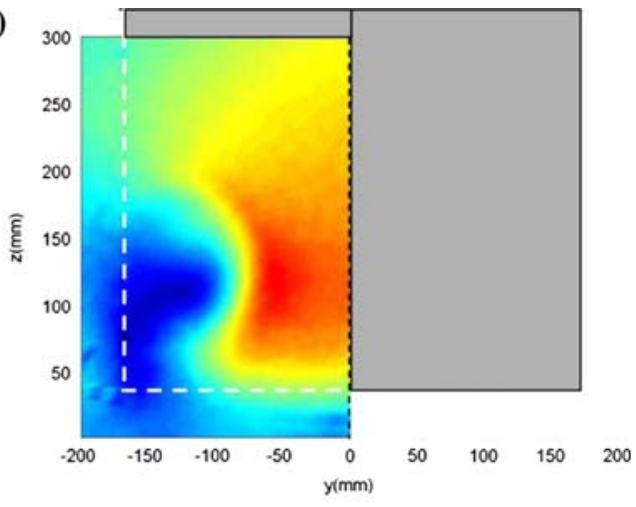

(d) vortices have opposite circulations). It is then possible to measure $\Gamma$ using Eq. 3:

$\Gamma=\oint_{\mathcal{C}} \vec{u} \cdot d \vec{l}=\int_{\mathcal{S}} \vec{\omega} \cdot d \vec{S}$

The corresponding results are displayed in Table 4 and reveal that the vortices intensity are arranged in the same order as the drag coefficient. Namely, for $s=0.140 \mathrm{~m}$ $\left(C_{d}=0.405\right)$ the circulation is higher than the reference, whereas for $s=0.20 \mathrm{~m}\left(C_{d}=0.280\right)$ it is lower. This suggests that the high drag case corresponds to strong longitudinal structures and short separation region, whereas the low drag case is related to weaker streamwise vortices and a large separation bubble.

\subsection{Discussion}

The previous results can be interpreted using the relation between the drag experienced by a body in a flow and its wake. Indeed, it is possible to evaluate the drag applied on a body in a stationary flow using the momentum conservation theorem applied on a finite domain containing the body and taking into account the pressure drop in the wake. It leads to the following definition for the drag force $F_{x}$ :

$F_{x}=\rho U_{0}^{2} \Sigma_{0}-\int_{\Sigma_{S}} \rho u^{2} \mathrm{~d} \sigma+\int_{\Sigma_{S}}\left(p_{0}-p\right) \mathrm{d} \sigma$

where $\Sigma_{S}$ and $\Sigma_{S}$ are upstream and downstream surface, respectively, of a volume containing the body, while $U_{0}$ and $p_{0}$ are respectively the freestream velocity and pressure and $u$ is the streamwise velocity measured in the downstream surface $\Sigma_{S}$. The mean drag then appears to be simply the sum of the momentum and the pressure deficits in the far wake.

In the case of simplified ground vehicles, or more generally 3D bluff-bodies Onorato et al. (1984) introduce the concept of "vortex drag" in which the contribution of the longitudinal vortices to the global drag is related to the rotational kinetic energy induced by these structures into the wake. It was confirmed later experimentally on a finitespan wing by Chometon and Laurent (1990). Onorato et al. (1984), corrected later by Ardonceau and Amani (1992), show that the drag force experienced by a vehicle can be written as: 
Fig. 20 PIV measurements in the vertical cross-section $x=580 \mathrm{~mm}$ (i.e. $130 \mathrm{~mm}$ downstream from the model) colored by the streamwise vorticity $\omega_{x}$. a location of the PIV plane. b reference flow $\left(C_{d}=0.315\right)$. $\mathbf{c}$ high drag case $\left(s=0.140 \mathrm{~m}\right.$ and $\left.C_{d}=0.405\right)$. d low drag case $(s=0.20 \mathrm{~m}$ and $C_{d}=0.280$ ). The PIV field is measured in one half of the vertical cross-section assuming symmetry for the mean velocity field (a)

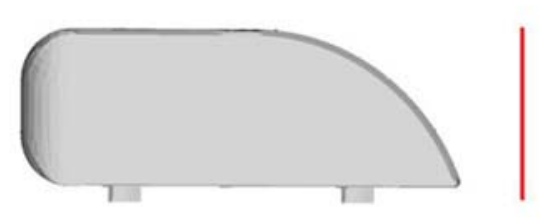

(b)

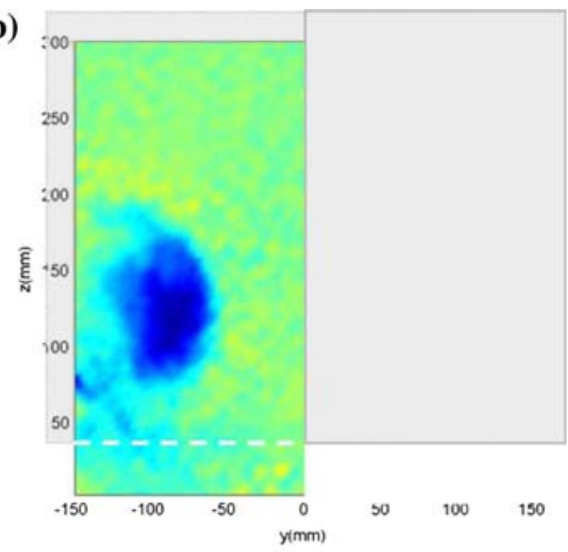

(d)

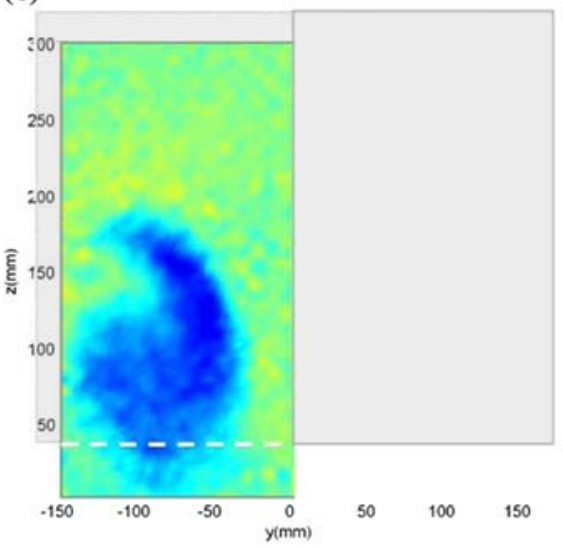

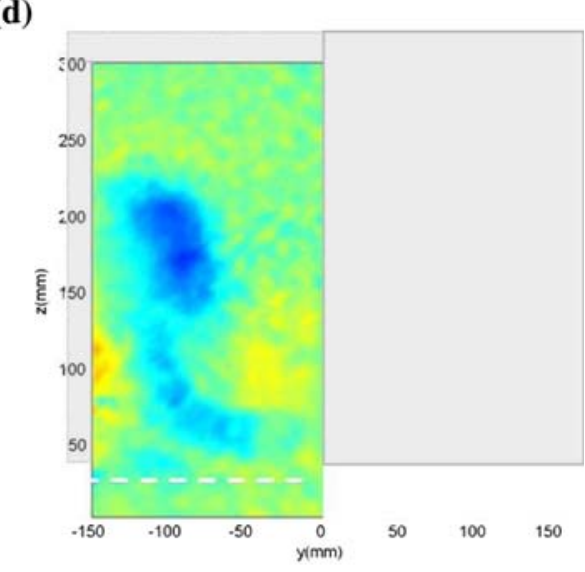

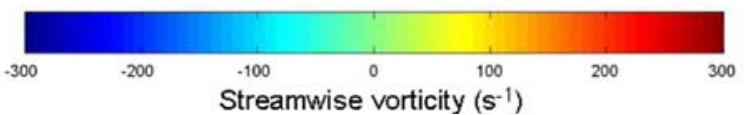

Table 4 Longitudinal vortex circulation $\Gamma\left(\mathrm{m}^{2} \mathrm{~s}^{-1}\right)$ evaluated from PIV measurements. Comparison between reference flow, high drag case and low drag case

\begin{tabular}{llll}
\hline & Reference flow & $s=0.18 \mathrm{~m}$ & $s=0.24 \mathrm{~m}$ \\
\hline$\Gamma\left(\mathrm{m}^{2} / \mathrm{s}\right)$ & 1.58 & 2.28 & 0.87 \\
\hline
\end{tabular}

$$
\begin{aligned}
F_{x}= & \frac{\rho U_{0}^{2}}{2} \int_{\Sigma_{S}}\left[1-\left(\frac{u}{U_{0}}\right)^{2}\right] \mathrm{d} \sigma \\
& +\frac{\rho U_{0}^{2}}{2} \int_{\Sigma_{S}}\left[\left(\frac{v}{U_{0}}\right)^{2}+\left(\frac{w}{U_{0}}\right)^{2}\right] \mathrm{d} \sigma+\int_{\Sigma_{S}}\left(p_{t 0}-p_{t}\right) \mathrm{d} \sigma
\end{aligned}
$$

where $p_{t}$ is the total pressure. The second term in Eq. 5 can clearly be interpreted as the contribution of the trailing vortices to the global drag or "vortex drag". The vortex drag then appears to be a simple extension of the induced drag, but in a more generic and well-suited formulation for 3D bluff-bodies. Finally, from a physical point of view one could even propose a slightly different analysis in which the pair of streamwise counter-rotating trailing vortices induces high transversal velocities between their cores, creating low pressure by simple Venturi effect. The downwash is then responsible for a non-negligible contribution to the drag through the pressure deficit.

The previously discussed notions concerning the contribution of streamwise structures to the total drag are formally equivalent but slightly differ from the conceptual point of view. But whatever the preferred terminology, it is important to underline the fact that the drag experienced by a 3D bluff-body includes contributions from the separation bubbles and from the trailing vortices, which both contribute to the total drag through the momentum and the pressure deficits. As a result, in order to reduce the total drag of a 3D bluff-body, one should aim at delaying separation and/or decreasing the intensity of the trailing vortices.

There are mainly two limitations to these models. First, in spite of the existence of many formulations to compute the drag from the velocity field, it is often very difficult to obtain experimentally the quantitative contributions of 
each terms, especially in complex 3D flows. Second, it is almost impossible to manipulate one flow structure without modifying the others when dealing with low aspect ratio 3D bluff-bodies such as the Ahmed body. The key point is then to focus on the global effects.

Together with the results presented in Sect. 4.4 and the previous discussion, a mechanism explaining the large drag and lift reduction can finally be proposed. The VGs located in the center induce an early separation of the boundary layer that modifies the balance between the recirculation bubble and the longitudinal vortices. The consequence is a modification of the characteristics of the trailing vortices, especially their circulation. When located at their optimal position, the VGs induce a reduction of the global streamwise circulation leading to a drag reduction. For the optimal configurations, the drag reduction induced by the weakening of the streamwise vortices becomes higher than the drag generation due to the larger recirculation bubble over the rear slant. The result is a global drag and lift reduction.

Finally, one can wonder about the efficiency of such a technique on a full-scale vehicle. As a matter of fact, the question of the scaling is difficult when dealing with vortex generators and requires dedicated studies. Nevertheless, one can give two tentative answers. First, the VGs presented in this study have been extensively tested on fullscale vehicles (Despré et al. 2003; Aider et al. 2009b). Their efficiency is recovered (in some cases improved) with slightly longer blades. Rounded VGs $\left(5 \times 10^{-3} \mathrm{~m}\right.$ radius), conform to safety rules for external devices, have also been tested and validated on real cars leading to the same drag and lift reductions. Second, the important scaling for the VGs is not based on the vehicle length but on the boundary layer thickness, which does not change drastically on a full scale model. Indeed, one has to consider a Reynolds number based on the height of the VG and the freestream velocity $\operatorname{Re}_{h}=U_{o} h / v$, or based on the boundary layer thickness $\operatorname{Re}_{\delta}=U_{o} \delta / v$, which is comparable between small and full scales experiments $\left(\operatorname{Re}_{\delta} \approx 10^{5}\right.$ for $U_{o}=40 \mathrm{~m} \mathrm{~s}^{-1}$ ).

\section{Conclusion}

An extensive parametric study of the influence of a vortex generators line on the aerodynamic coefficients of a 3D bluff-body has been carried out. Both the geometry of the VGs (small trapezoidal blades) and of the bluff-body (a modified Ahmed body) are non-conventional. The line of vortex generators appears to be very efficient for drag $(-12 \%)$ and lift reduction (more than $-60 \%)$. For some configurations, the lift on the rear axle can even be cancelled out (more than $-100 \%$ ). A strong dependance on most of the geometrical parameters defining the line of vortex generators is also found. For instance, a clear evolution of the drag and lift as a function of the longitudinal position of the VG line has been shown, with a welldefined minimum for a position upstream of the natural separation line. When focusing on two given angles for the VGs, both configurations exhibit different dependencies on longitudinal position and transversal spacing. Finally, a new set-up with motorized VGs is presented. Thanks to these mechanical vortex generators, the optimal configurations for both drag and lift can be found more easily in the space parameter $(s, \alpha)$. The importance of both the location of the VGs and of the angle between the blade and the wall is clearly confirmed for both drag and lift. It is also an important step toward a closed-loop experiment (Beaudoin et al. 2006) as the motorization is not only a trick for remotely changing the angle between the GVs and the wall: the angle between the blade and the wall can now be used as a dynamic parameter in a closed-loop experiment. In such a configuration, the angle of the VGs changes as a function of a given input, which can be the freestream velocity (Beaudoin et al. 2008).

To get a better understanding of the interactions between the VGs and the overall flow structure, the velocity field in the near-wake of the bluff-body for three different configurations are compared. An experimental investigation of the three-dimensional time-averaged flow reveals that the drag and lift reduction is closely related to the reduction of the circulation of the streamwise vortices arising on the side edges of the rear part of the model. It appears that the vortex generators trigger an early separation leading to a larger recirculation bubble over the rear slant and a breakdown of the balance between the separation bubble and the longitudinal vortices. It also leads to a modification of their relative contributions to the global drag through momentum and pressure deficits. As a result, a drag reduction is clearly associated with a large separation region and weak streamwise vortices. It is then demonstrated that triggering early separation can be a very efficient way to reduce the total drag of a bluff-body, specifically when the trailing vortices and the recirculation bubble interact in the near-wake.

Acknowledgments We would like to thank G. Hulin, A. Lebau for their contribution to the wind tunnel measurements.

\section{References}

Ahmed SR (1983) Influence of base slant on the wake structure and drag of road vehicles. J Fluids Eng 105:429-434

Ahmed SR, Ramm R, Faltin G (1984) Some salient features of the time-averaged ground vehicle wake. SAE Paper pp 1-30, 840300

Aider JL, Beaudoin JF (2008) Drag and lift reduction of a 3d bluffbody using flaps. Exp Fluids 44(4):491-501 
Aider J-L, Beaudoin J-F, Wesfreid JE (2009a) Investigation of the flow around bluff-body vortex generators. Application to the control of the drag and lift of a 3d bluff-body. Exp Fluids (In preparation)

Aider J-L, Lasserre J-J, Beaudoin J-F, Herbert V, Wesfreid JE (2009b) Contrôle d'écoulement en aérodynamique automobile. In Actes du Congrès Français de Mécanique (CFM'09). Marseille, France

Aider JL, Lasserre JJ, Herbert V (2003) Dispositif aerodynamique pour un vehicule automobile - Aerodynamic system for road vehicles. Patent FR 0305923 - EP 04 292001.7. PSA PeugeotCitroën

Andersson P, Brandt L, Bottaro A, Henningson DS (2002) On the breakdown of boundary layer streaks. J Fluid Mech 428:29-60

Angele K, Grewe F (2002) Streamwise vortices in turbulent boundary layer separation control. In: Proceeding of the 11th symposium on application of laser techniques to fluid mechanics. Lisbon, Portugal

Ardonceau P, Amani G (1992) Remarks on the relation between lift induced drag and vortex drag. Eur J Mech B/Fluids 11(4):455460

Beaudoin JF, Cadot O, Aider JL, Gosse K, Paranthoën P, Hamelin B, Tissier M, Allano D, Mutabazi I, Gonzales M, Wesfreid JE (2004) Cavitation as a complementary tool for automotive aerodynamics. Exp Fluids 37(5):763-768

Beaudoin JF, Cadot O, Aider JL, Wesfreid JE (2006) Drag reduction of a bluff-body using adaptive control methods. Phys Fluids 18(085107): 10

Beaudoin JF, Cadot O, Aider JL, Wesfreid JE (2008) Drag reduction of a $3 \mathrm{~d}$ bluff-body by closed loop control using oscillating vortex generators and wall pressure measurement. In: Morrison $\mathrm{J}$, Jonathan F, Birch DM, Lavoie P (eds) Proceedings of the IUTAM symposium on flow control and MEMS, London, United-Kingdom, 2006 IUTAM Bookseries, vol. 7. Springer

Betterton JG, Hackett HC, Ashill PR, Wilson MJ, Woodcock IJ, Tilman CP, Langan KJ (2000) Laser doppler anemometry investigation on sub boundary layer vortex generators for flow control. In: Proceeding of the 10th symposium on application of laser techniques to fluid mechanics. Lisbon, Portugal

Bewley TR, Moin P, Temam R (2001) DNS-based predictive control of turbulence: an optimal benchmark for feedback algorithms. J Fluid Mech 447:179-225

Chometon F, Laurent J (1990) Study of three dimensional separated flows, relation between induced drag and vortex drag. Eur J Mech B/Fluids 9(5):437-455

Chun S, Lee I, J Sung H (1999) Effect of spanwise-varying local forcing on turbulent separated flow over a backward-facing step. Exp Fluids 26:437-440

Dalton C, Xu Y, Owen JC (2001) The suppression of lift on a circular cylinder due to vortex shedding at moderate Reynolds numbers. J Fluids Struc 15:617-628

Despré C, Colin O, Lasserre J-J, Aider JL (2003) Validation du potentiel de générateurs de vortex actifs pour la réduction de tranée et de portance d'une Citroën $\mathrm{C} 4$ berline ou coupée. Technical Reports. PSA Peugeot-Citroën

Duriez T, Aider J-L, Wesfreid JE (2006) Base flow modification by streamwise vortices. Application to the control of separated flows. In Control of Separated Flows, ASME technical paper FEDSM2006-98541. Miami, USA

Duriez T, Aider J-L, Wesfreid JE (2008) Linear modulation of a boundary layer induced by vortex generators. In: 4th Flow control conference, AIAA technical paper AIAA-2008-4076. Seattle, USA
Duriez T, Aider J-L, Wesfreid JE (2009) Experimental evidence of a self-sustaining process through streaks generation in a flat plate boundary layer. Phys Rev Lett to be published

Gad-El-Hak M, Bushnell DM (1991) Separation control: review. J Fluids Eng 113:5-30

Giannetti F, Luchini P (2007) Structural sensitivity of the first instability of the cylinder wake. J Fluid Mech 581:167-197

Gilliéron P, Chometon F (1999) Modelling of stationary threedimensional separated air flows around an ahmed reference model. ESAIM: Proc 7:173-182

Godard G, Stanislas M (2006) Control of a decelerating boundary layer. part 1: Optimization of passive vortex generators. Aerosp Sci Technol 10:181-191

Golhke M, Beaudoin JF, Amielh M, Anselmet F (2008) Experimental analysis of flow structures and forces on a 3d-bluff-body in constant cross-wind. Exp Fluids 43(4):579-594

Greenblatt D, Wygnanski IJ (2000) The control of flow separation by periodic excitation. Prog Aerosp Sci 36:487-545

Hucho WH (1998) Aerodynamics of road vehicles. Cambridge University Press, Cambridge

Joslin RD (1998) Aircraft laminar flow control. Annu Rev Fluid Mech 30:1-29

Kim J (2003) Control of turbulent boundary layers. Phys Fluids 15(5):1093-1105

Leclerc C, Levalois E, Gilliéron P, Kourta A (2006) Aerodynamic drag reduction by synthetic jet: 2D numerical study around simplified car. In 3rd AIAA flow control conference, 5-8 June 2006, San Francisco, CA, USA. AIAA Paper 2006-3337

Lin C, Chiu PH, Shieh SJ (2002) Characteristics of horseshoe vortex system near a vertical plate-base juncture. Exp Therm Fluid Sci $27: 25-46$

Lin J (2002) Review of research on low-profile vortex generators to control boundary-layer separation. Prog Aerosp Sci 38:389-420

Onorato M, Costelli AF, Garrone A (1984) Drag measurement through wake analysis. SAE Paper pp 85-93, 840302

Raffel M, Willert C, Wereley S, Kompenhans J (2007) Particle image velocimetry. A practical guide. Second Edition. Springer, Berlin

Roumeas M, Gillieron P, Kourta A (2009) Analysis and control of the near-wake flow over a square-back geometry. Comput Fluids 38(1):60-70

Simpson RL (2001) Junction flows. Annu Rev Fluid Mech 33:415443

Smith FT (1994) Theoretical prediction and design for vortex generators in turbulent boundary layers. J Fluid Mech 270:91131

Song S, Eaton JK (2002) The effects of wall roughness on the separated flow over a smoothly contoured ramp. Exp Fluids 33:38-46

Spohn A, Gilliéron P (2002) Flow separations generated by a simplified geometry of an automotive vehicle. In: Proceeding of the IUTAM symposium on unsteady separated flows. Toulouse, France

Strykowski PJ, Sreenivasan KR (1990) On the formation and suppression of vortex shedding at low reynolds number. J Fluid Mech 218:71-107

Verzicco R, Fatica M, Iaccarino G, Moin P, Khalighi B (2002) Large eddy simulation of a road vehicle with drag-reduction devices. AIAA J 40(12):2447-2455

Vino G, Watkins S, Mousley P, Watmuff J, Prasad S (2005) Flow structures in the near-wake of the ahmed model. J Fluids Struc 20(5):673-695 